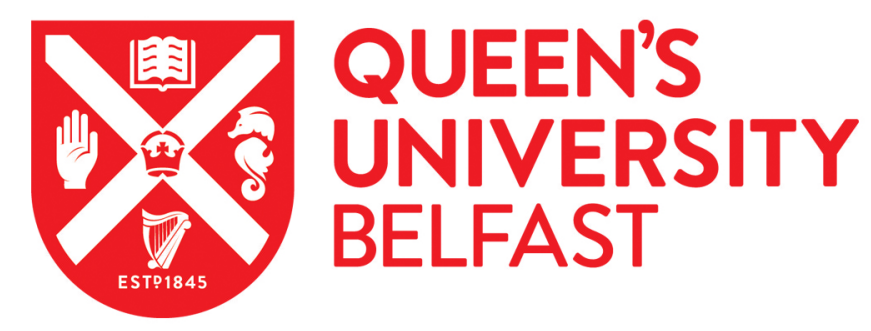

\title{
Optimization for Signal Transmission and Reception in a Macrocell of Heterogeneous Uplinks and Downlinks
}

\author{
Yu, H., Tuan, H. D., Duong, T. Q., Poor, H. V., \& Fang, Y. (2020). Optimization for Signal Transmission and \\ Reception in a Macrocell of Heterogeneous Uplinks and Downlinks. IEEE Transactions on Communications. \\ https://doi.org/10.1109/TCOMM.2020.3014945
}

\author{
Published in: \\ IEEE Transactions on Communications
}

\section{Document Version:}

Peer reviewed version

Queen's University Belfast - Research Portal:

Link to publication record in Queen's University Belfast Research Portal

\section{Publisher rights}

(c) 2020 IEEE.

This work is made available online in accordance with the publisher's policies. Please refer to any applicable terms of use of the publisher.

\section{General rights}

Copyright for the publications made accessible via the Queen's University Belfast Research Portal is retained by the author(s) and / or other copyright owners and it is a condition of accessing these publications that users recognise and abide by the legal requirements associated with these rights.

Take down policy

The Research Portal is Queen's institutional repository that provides access to Queen's research output. Every effort has been made to ensure that content in the Research Portal does not infringe any person's rights, or applicable UK laws. If you discover content in the Research Portal that you believe breaches copyright or violates any law, please contact openaccess@qub.ac.uk. 


\title{
Optimization for Signal Transmission and Reception in a Macrocell of Heterogeneous Uplinks and Downlinks
}

\author{
H. Yu ${ }^{1,2}$, H. D. Tuan ${ }^{2}$, T. Q. Duong ${ }^{3}$, H. V. Poor ${ }^{4}$, and Y. Fang ${ }^{1}$
}

\begin{abstract}
Internet-of-things (IoT) applications continue to drive advancements in serving as many heterogeneous downlinks and uplinks of low-latency as possible within a constrained communication bandwidth. Full-duplexing (FD) transceivers have been introduced to implement simultaneous signal transmission and reception (STR) over the whole frequency band. However, both inter-link interference and FD loop-interference are hardly suppressed to a necessary level for the effectiveness of the FD-based STR even for microcells. The paper proposes an alternative STR technique per one time-slot for macrocells, where a fraction of a time-slot is used for downlinks and the remaining complementary fraction of the time-slot is used for uplinks. Thus, STR over the whole bandwidth can be implemented in a way with no loop interference. Furthermore, an another approach of using a fraction of the whole bandwidth for downlinks and the remaining complementary fraction of the bandwidth for uplinks over the whole time-slot is also proposed. The problem of both downlink and uplink beamforming to maximize the energy efficiency of such heterogeneous networks subject to the quality-of-service in terms of downlink and uplink throughput is examined for all three possible STRs. Numerical results demonstrate the advantages of the time-fraction-wise STR and bandwidth-fraction-wise STR over the FD-based STR, where the time-fraction-wise STR is not only the best in serving the same numbers of downlinks and uplinks but also is capable of serving many more downlinks and uplinks with a higher energy efficiency.
\end{abstract}

Index Terms-Heterogeneous networks, signal transmission and reception, energy efficiency, fractional time-slot allocation, fractional bandwidth allocation, downlink and uplink beamforming, nonconvex optimization

\section{INTRODUCTION}

\footnotetext{
${ }^{1}$ School of Communication and Information Engineering, Shanghai University, Shanghai, China (email: hongwenyu@ shu.edu.cn, yfang@staff.shu.edu.cn)

${ }^{2}$ School of Electrical and Data Engineering, University of Technology Sydney, Broadway, NSW 2007, Australia (email: hongwen.yu@student.uts.edu.au; Tuan.Hoang@uts.edu.au)

${ }^{3}$ School of Electronics, Electrical Engineering and Computer Science, Queen's University Belfast, Belfast BT7 1NN, UK (email: trung.q.duong@qub.ac.uk)

${ }^{4}$ Department of Electrical Engineering, Princeton University, Princeton, NJ 08544, USA (email: poor@princeton.edu)

This work was supported in part by the National Natural Science Foundation of China (NSFC) under Grant 61901254 and Grant 61673253, in part by the Institute for Computational Science and Technology, Hochiminh City, Vietnam, in part by the Australian Research Councils Discovery Projects under Grant DP190102501, in part by the U.K. Royal Academy of Engineering Research Fellowship under Grant RF1415 \14\22, and in part by the U.S. National Science Foundation under Grant CCF-0939370 and Grant CCF1908308.
}

$\mathbf{T}$ HE Internet-of-things (IoT) is characterized by massive wireless connectivity of low latency, which means that the future wireless communication must be cost-efficient and environment-friendly in accommodating a plethora of wireless downlinks and uplinks over the same time slot within a constrained communication bandwidth [1]. One of the first and perhaps the most natural answers is the full-duplexing (FD) exploitation, which provides means for simultaneous signal transmission and reception (STR) over the same frequency band/bandwidth by the same base station (BS) of colocated transmit and receive antennas [2]-[4]. However, the FD exploitation suffers its self-interference (SI) bottleneck as the interference to the receive signal from the transmit signal is expected very strong [5], [6]. Even for microcells, it is impossible to control SI under a level so that the FDbased STR is more spectral-efficient or energy-efficient than the conventional half-duplexing (HD)-based transmission and reception, which transmit and receive signal in two separated time-slots, even by using the advanced techniques of signal processing [7]-[19]. Motivated by this unsolved issue of the FD exploitation, and also originated from [20], where the information and energy are proposed to be transferred separately over the same wireless channels within a time-slot instead of their simultaneous transfer, it was suggested in [19], [21], [22] to use a fraction of a time-slot to transmit signals to the relays and then the remaining complementary timefraction to forward the received signals at the relays to the destination end. Such time-fraction-wise half-duplexing (HD) relaying is not only much easier implemented but is much better than that using the FD-based relaying. Additionally, unlike FD-based relaying, which must use a half of the relays' antennas for receiving signals and another half of relays' antennas for forwarding them, the time-fraction-wise HD relaying can use all available antennas for receiving and then forwarding signals to enjoy multi-antenna diversity in both signal transmission and reception. The benefit of fractional time allocations in terms of improving the capacity of singleuser and single-input single output HD decode-and-forward systems has been shown earlier e.g. in [23], [24]. Another approach to STR within a time-slot, which can also avoid the SI, is to transmit signal and receive signal at the same time-slot using separated communication bandwidths, i.e. the whole bandwidth is divided into two parts, one is used for signal transmission and the remaining complementary one is used for signal reception. This bandwidth-fraction-wise STR must still use a half of antennas for signal transmission and 
another half of antennas for signal reception. Moreover, it can be practically implemented only when the bandwidth parts are fixed beforehand.

The present paper examines the three aforementioned approaches to serve a macrocell of heterogeneous downlinks and uplinks within a time-slot. Under the FD-based STR, the focus is to design the transmit beamformers at the BS and uplink users. Under the time-fraction-wise STR, the focus is to jointly design the time-fraction-wise beamformers at the BS and uplink users and time-fraction allocation, while the focus under the bandwidth-fraction-wise STR is to jointly design the bandwidth-fraction-wise beamformers at the BS and uplink users and bandwidth-fraction allocation. Motivated by saving energy to control the negative impact of wireless communication in global warming [25], [26], all these design problems aim to maximize the network energy-efficiency (EE) under the quality-of-service (QoS) constraints in terms of downlink and uplink throughput. While the spectral efficiency (SE) of (microcell) FD transceivers has been considered previously (see e.g. [10] and references therein), its EE subject to the downlink and uplink QoS constraints was not appropriately considered. This paper is the first work to consider STR over macrocell, which exploits time-fraction-wise and bandwidth-fraction-wise beamforming. All the problems of beamforming design are formulated as nonconcave objective function optimization subject to nonconvex constraints, which are computationally challenging. Our further contributions are developments of tailored path-following algorithms for their computation.

The rest of the paper is organized as follows. Section II is devoted to the problem of EE maximization under FDbased STR, where in the end the FD-based STR's drawbacks are analysed in depth. Sections III and IV then are devoted to the problem of EE maximization under time-fraction-wise STR and bandwidth-fraction-wise STR, which provide remedy means for the FD-based STR. Simulations are provided in Section $\mathrm{V}$ to substantiate the analytical development of the previous sections, where the SE of STR is also additionally revealed. The appendix provides some fundamental inequalities used for deriving the equations in Sections II-IV.

Notation. All variables are boldfaced. $I_{n}$ is the identity matrix of size $n \times n$. $\mathbb{E}[$.$] denotes the expectation operator$ and $\Re\{$.$\} denotes the real part of a complex number. \left(v_{i}\right)_{i \in D}$ $\left(\left(v_{i}\right)_{i=1}^{n}\right.$, resp. $)$ is the arrangement for the vectors $v_{i}, i \in D$ $(i=1, \ldots, n$, resp.) in a single vector.

\section{FD-BASED STR}

Consider a macrocell of a BS serving $d$ downlink singleantenna users termed by $\mathrm{DL}_{j}, j=1, \ldots, d$, and $u$ uplink single-antenna users termed by $\mathrm{UL}_{i}, i=1, \ldots, u$, as illustrated by Fig. 1. The BS is equipped with $n$ antennas. Under the FD-based STR, $n_{1}$ antennas are used for signal transmission to serve the downlinks and other $n_{2}=n-n_{1}$ antennas are used for signal reception to serve the uplinks. Usually, $n_{1} \geq d$ and $n_{2} \geq u$ are required for practical implementation. Let $s_{j}^{\mathrm{d}} \in \mathbb{C}$ with $\left|s_{j}^{\mathrm{d}}\right|=1$ be the information intended for $\mathrm{DL}_{j}$, which is beamformed by $\boldsymbol{v}_{j} \in \mathbb{C}^{n_{1}}$ before

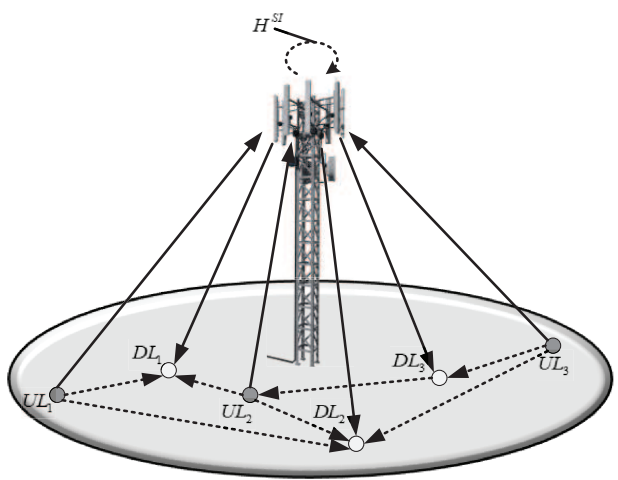

Fig. 1: FD-based STR system

the BS's transmission. Let $s_{i}^{\mathrm{u}} \in \mathbb{C}$ with $\left|s_{i}^{\mathrm{u}}\right|=1$ be the information $\mathrm{UL}_{i}$ intends to send to the $\mathrm{BS}$, which is allocated by power $\boldsymbol{p}_{i}^{2}$ before the uplinks' transmission. For notational convenience, define

$$
\begin{gathered}
\boldsymbol{v} \triangleq\left\{\boldsymbol{v}_{j}: j=1, \ldots, d\right\} \\
\boldsymbol{p} \triangleq\left\{\boldsymbol{p}_{i}: i=1, \ldots, u\right\}, \mathcal{D}=\{1, \ldots, d\} .
\end{gathered}
$$

The received signal at $\mathrm{DL}_{j}$ is

$$
\begin{aligned}
y_{j} \triangleq \underbrace{h_{j, \mathrm{bs}}^{H} \boldsymbol{v}_{j} s_{j}^{\mathrm{d}}}_{\text {desired signal }} & +\underbrace{\sum_{\ell \in \mathcal{D} \backslash\{j\}} h_{j, \mathrm{bs}}^{H} \boldsymbol{v}_{\ell} s_{\ell}^{\mathrm{d}}}_{\mathrm{DL} \text { interference }}+\underbrace{\sum_{i=1}^{u} h_{j, i} \boldsymbol{p}_{i} s_{i}^{\mathrm{u}}}_{\text {UL interference }} \\
& +n_{j}, \quad j=1, \ldots, d,
\end{aligned}
$$

where $h_{j, \text { bs }} \in \mathbb{C}^{n_{1}}$ and $h_{j, i} \in \mathbb{C}$ are the channels from the BS to $\mathrm{DL}_{j}$ and from $\mathrm{UL}_{i}$ to $\mathrm{DL}_{j}$, respectively, and $n_{j}$ is the background white Gaussian noise with the variance $\sigma_{\mathrm{d}}^{2}$.

The throughput at $\mathrm{DL}_{j}$ is

$$
r_{j}^{\mathrm{d}}(\boldsymbol{v}, \boldsymbol{p}) \triangleq \ln \left(1+\frac{\left|\lambda_{j}\left(\boldsymbol{v}_{j}\right)\right|^{2}}{\psi_{j}(\boldsymbol{v}, \boldsymbol{p})}\right),
$$

where $\lambda_{j}\left(\boldsymbol{v}_{j}\right) \triangleq h_{j, \mathrm{bs}}^{H} \boldsymbol{v}_{j}, \quad$ and $\psi_{j}(\boldsymbol{v}, \boldsymbol{p}) \triangleq$ $\sum_{\ell \in \mathcal{D} \backslash\{j\}}\left|h_{j, \mathrm{bs}}^{H} \boldsymbol{v}_{\ell}\right|^{2}+\sum_{i=1}^{u} \boldsymbol{p}_{i}^{2}\left|h_{j, i}\right|^{2}+\sigma_{\mathrm{d}}^{2}$, which is a convex quadratic function. At the same time, the received signal at the $\mathrm{BS}$ is

$$
\begin{aligned}
& y_{b s} \triangleq \underbrace{\sum_{i=1}^{u} h_{\mathrm{bs}, i} \boldsymbol{p}_{i} s_{i}^{\mathrm{u}}}_{\text {desired signal }}+\underbrace{H^{\mathcal{S I}} \sum_{j=1}^{d} \boldsymbol{v}_{j} \tilde{s}_{j}}_{\text {residual SI }}+n_{\mathrm{bs}} \\
& =\left[\begin{array}{llll}
h_{\mathrm{bs}, 1} & h_{\mathrm{bs}, 2} & \ldots & h_{\mathrm{bs}, u}
\end{array}\right]\left[\begin{array}{c}
\boldsymbol{p}_{1} s_{1}^{\mathrm{u}} \\
\boldsymbol{p}_{2} s_{2}^{\mathrm{u}} \\
\ldots \\
\boldsymbol{p}_{u} s_{u}^{\mathrm{u}}
\end{array}\right]+\tilde{n}_{\mathrm{bs}},
\end{aligned}
$$

where $h_{\mathrm{bs}, i} \in \mathbb{C}^{n_{2}}$ is the channel vector from $\mathrm{UL}_{i}$ to the $\mathrm{BS}$, $n_{\text {bs }}$ is the background white Gaussian noise with the variance $\sigma_{\text {bs }}^{2} I_{n_{2}}$. Furthermore, $H^{\mathcal{S I}} \in \mathbb{C}^{n_{2} \times n_{1}}$ is the residual self-loop channel from the transmit antennas to the receive antennas at the BS after all real-time cancelation in both analog and digital domains [27], [28]. $\tilde{s}_{j}$ is the additive Gaussian noise with $\mathbb{E}\left[\left|\tilde{s}_{j}\right|^{2}\right]=\sigma_{S I}^{2}$ that models the effect of analog circuit 
non-ideality and the limited dynamic range of the analog-todigital converter (ADC), so the SI level $\sigma_{S I}^{2}$ is the ratio of the average SI powers before and after the SI cancelation process [5], [27], [29], [30]. The term $\tilde{n}_{\text {bs }} \triangleq H^{\mathcal{S I}} \sum_{j=1}^{d} \boldsymbol{v}_{j} \tilde{s}_{j}+n_{\text {bs }}$ in (4) represents the self-loop interference plus noise. By assuming that the entries of the self-loop channel $H^{\mathcal{S I}}$ in (3) are treated as independent circularly symmetric complex Gaussian random variables with zero mean and unit variance, $\tilde{n}_{\text {bs }}$ can be assumed white noise with the covariance

$$
\left(\sigma_{S I}^{2} \sum_{j=1}^{d}\left\|\boldsymbol{v}_{j}\right\|^{2}+\sigma_{\text {bs }}^{2}\right) I_{n_{2}}
$$

which only depends on the BS transmit power and thus cannot be mitigated by the transmit beamformer $\boldsymbol{v}$.

Make QR decomposition

$$
\left[\begin{array}{llll}
h_{\mathrm{bs}, 1} & h_{\mathrm{bs}, 2} & \ldots & h_{\mathrm{bs}, u}
\end{array}\right]=Q_{\mathrm{bs}} R_{\mathrm{bs}} \in \mathbb{C}^{n_{2} \times u},
$$

where $Q_{\mathrm{bs}} \triangleq\left[\begin{array}{llll}q_{\mathrm{bs}, 1} & q_{\mathrm{bs}, 2} & \ldots & q_{\mathrm{bs}, n_{2}}\end{array}\right] \in \mathbb{C}^{n_{2} \times n_{2}}$ is an unitary matrix and $R_{\mathrm{bs}} \in \mathbb{C}^{n_{2} \times u}$ is an upper rectangular matrix. It follows from (4) that

$$
Q_{\text {bs }}^{H} y_{\text {bs }}=R_{\text {bs }}\left[\begin{array}{c}
\boldsymbol{p}_{1} s_{1}^{\mathrm{u}} \\
\boldsymbol{p}_{2} s_{2}^{\mathrm{u}} \\
\ldots \\
\boldsymbol{p}_{u} s_{u}^{\mathrm{u}}
\end{array}\right]+Q_{\mathrm{bs}}^{H} \tilde{n}_{\text {bs }} .
$$

Upon successive interference cancellation (SIC) decoding, the throughput of $s_{i}^{\mathrm{u}}$ at the BS is

$$
r_{i}^{\mathrm{u}}\left(\boldsymbol{v}, \boldsymbol{p}_{i}\right)=\ln \left(1+\frac{\boldsymbol{p}_{i}^{2}\left|R_{\mathrm{bs}}(i, i)\right|^{2}}{\psi_{\mathrm{bs}}(\boldsymbol{v})}\right), \quad i=1, \ldots, u,
$$

where $\psi_{\text {bs }}(\boldsymbol{v}) \triangleq \sigma_{S I}^{2} \sum_{j \in \mathcal{D}}\left\|\boldsymbol{v}_{j}\right\|^{2}+\sigma_{\text {bs }}^{2}$.

Following e.g. [31], the consumed power $P^{\text {tot }}$ for the STR is modelled by the following convex quadratic function

$$
P^{t o t}(\boldsymbol{v}, \boldsymbol{p})=\zeta P^{t}(\boldsymbol{v}, \boldsymbol{p})+P_{B S}+P_{U E},
$$

where $P^{t}(\boldsymbol{v}, \boldsymbol{p}) \triangleq \sum_{j \in \mathcal{D}}\left\|\boldsymbol{v}_{j}\right\|^{2}+\sum_{i=1}^{u} \boldsymbol{p}_{i}^{2}$ is the total transmit power of the BS and UEs, and $\zeta, P_{B S}$ and $P_{U E}$ are the reciprocal of drain efficiency of power amplifier, the circuit power of the BS and UEs, respectively.

We consider the following design problem of downlink beamforming and uplink power allocation to maximize the FD-based STR's EE:

$$
\begin{gathered}
\max _{\boldsymbol{v}, \boldsymbol{p}} \frac{\sum_{j=1}^{d} r_{j}^{\mathrm{d}}(\boldsymbol{v}, \boldsymbol{p})+\sum_{i=1}^{u} r_{i}^{\mathrm{u}}\left(\boldsymbol{v}, \boldsymbol{p}_{i}\right)}{P^{t o t}(\boldsymbol{v}, \boldsymbol{p})} \\
\text { s.t. } \quad \sum_{j=1}^{d}\left\|\boldsymbol{v}_{j}\right\|^{2} \leq P_{\mathrm{BS}}^{\max }, \\
0<\boldsymbol{p}_{i} \leq \sqrt{P_{\mathrm{UE}}^{\max }}, \quad i=1, \ldots, u, \\
r_{j}^{\mathrm{d}}(\boldsymbol{v}, \boldsymbol{p}) \geq r_{\mathrm{d}}, \quad j=1, \ldots, d, \\
r_{i}^{\mathrm{u}}\left(\boldsymbol{v}, \boldsymbol{p}_{i}\right) \geq r_{\mathrm{u}}, \quad i=1, \ldots, u,
\end{gathered}
$$

where (10b)-(10c) cap the transmit power constraints, while (10d)-(10e) cap the QoS constraints for both downlink and uplink transmission because $r_{\mathrm{d}}$ and $r_{\mathrm{u}}$ are throughput thresholds. $P_{\mathrm{BS}}^{\max }$ and $P_{\mathrm{UE}}^{\max }$ are the power budget for the $\mathrm{BS}$ and $\mathrm{UL}_{i}$, respectively.

By changing variable if necessary one can replace $\left|\lambda_{j}\left(\boldsymbol{v}_{j}\right)\right|^{2}$ by $\left(\bar{\lambda}_{j}\left(\boldsymbol{v}_{j}\right)\right)^{2}$ for $\bar{\lambda}_{j}\left(\boldsymbol{v}_{j}\right)=\Re\left\{\lambda_{j}\left(\boldsymbol{v}_{j}\right)\right\}$ in (2), so

$$
r_{j}^{\mathrm{d}}(\boldsymbol{v}, \boldsymbol{p})=\ln \left(1+\frac{\left(\bar{\lambda}_{j}\left(\boldsymbol{v}_{j}\right)\right)^{2}}{\psi_{j}(\boldsymbol{v}, \boldsymbol{p})}\right),
$$

and the downlink QoS constraint (10d) is equivalent to the second-order cone (SOC) constraint

$$
\begin{aligned}
\bar{\lambda}_{j}\left(\boldsymbol{v}_{j}\right) \geq & \sqrt{e^{r_{\mathrm{d}}}-1} \sqrt{\psi_{j}(\boldsymbol{v}, \boldsymbol{p})} \\
= & \sqrt{e^{r_{\mathrm{d}}}-1}\left\|\begin{array}{c}
\left(h_{j, \mathrm{bs}}^{H} \boldsymbol{v}_{\ell}\right)_{\ell \in \mathcal{D} \backslash\{j\}} \\
\left(\boldsymbol{p}_{i}\left|h_{j, i}\right|\right)_{i=1}^{u} \\
\sigma_{\mathrm{d}}
\end{array}\right\|_{2}, \\
& j=1, \ldots, d,
\end{aligned}
$$

while the uplink QoS constraint (10e) is also equivalent to the following SOC constraint

$$
\begin{aligned}
\boldsymbol{p}_{i}\left|R_{\mathrm{bs}}(i, i)\right| \geq & \sqrt{e^{r_{\mathrm{u}}}-1} \sqrt{\psi_{\mathrm{bs}}(\boldsymbol{v})} \\
= & \sigma_{S I} \sqrt{e^{r_{\mathrm{u}}}-1}\left\|\begin{array}{c}
\left(\boldsymbol{v}_{i}\right)_{i=1}^{d} \\
\sigma_{\mathrm{bs}} / \sigma_{S I}
\end{array}\right\|_{2}, \\
& i=1, \ldots, u .
\end{aligned}
$$

Therefore, the problem (10) is equivalent to the following convex constrained optimization problem

$$
\begin{gathered}
\max _{\boldsymbol{v}, \boldsymbol{p}} \Phi(\boldsymbol{v}, \boldsymbol{p}) \triangleq \frac{\sum_{j=1}^{d} r_{j}^{\mathrm{d}}(\boldsymbol{v}, \boldsymbol{p})+\sum_{i=1}^{u} r_{i}^{\mathrm{u}}\left(\boldsymbol{v}, \boldsymbol{p}_{i}\right)}{P^{t o t}(\boldsymbol{v}, \boldsymbol{p})} \\
\text { s.t. } \quad(10 b),(10 c),(11),(12),
\end{gathered}
$$

where the computational difficulty is concentrated at its objective function $\Phi(\boldsymbol{v}, \boldsymbol{p})$, which is not concave, making (13) a nonconvex problem. Usually, this objective is handled by fractional programming. Let $\left(v^{(\kappa)}, p^{(\kappa)}\right)$ be a feasible point for $(10)$ found from the $(\kappa-1)$ th iteration. Then the so called Dinkelbach's iteration [32] invokes the following optimization problem to generate $\left(v^{(\kappa+1)}, p^{(\kappa+1)}\right)$ at the $\kappa$-th iteration: $\left(v^{(\kappa+1)}, p^{(\kappa+1)}\right)$ at the $\kappa$-th iteration:

$$
\begin{aligned}
\max _{\boldsymbol{v}, \boldsymbol{p}} & \sum_{j=1}^{d} r_{j}^{\mathrm{d}}(\boldsymbol{v}, \boldsymbol{p})+\sum_{i=1}^{u} r_{i}^{\mathrm{u}}\left(\boldsymbol{v}, \boldsymbol{p}_{i}\right)-\Phi\left(v^{(\kappa)}, p^{(\kappa)}\right) P^{t o t}(\boldsymbol{v}, \boldsymbol{p}) \\
\text { s.t. } & (10 b),(10 c),(11),(12) .
\end{aligned}
$$

However, this problem is still nonconvex and thus computationally intractable as the first two terms in its objective function, which constitute the numerator of the objective function $\Phi(\boldsymbol{v}, \boldsymbol{p})$ in (13) are not concave. Our previous works [19], [33] have proposed to optimize the fractional objective function $f(\boldsymbol{v}, \boldsymbol{p})$ in (12), avoiding the computational intractable iteration (14). We now develop another path-following iterations, which like (14) generate a sequence $\left\{f\left(v^{(\kappa)}, p^{(\kappa)}\right)\right\}$ of improved values of the objective in (12) but in contrast to (14) invoke a simple quadratic optimization problem at each iteration and thus are very computationally efficient. 
Applying the inequality (73) in the appendix for $\boldsymbol{x}=$ $\bar{\lambda}_{j}\left(\boldsymbol{v}_{j}\right), \boldsymbol{y}=\psi_{j}^{\mathrm{d}}(\boldsymbol{v}, \boldsymbol{p})$ and $\bar{x}=\bar{\lambda}_{j}\left(v_{j}^{(\kappa)}\right), \bar{y}=\psi_{j}^{\mathrm{d}}\left(v^{(\kappa)}, p^{(\kappa)}\right)$ yields

$$
\begin{aligned}
r_{j}^{\mathrm{d}}(\boldsymbol{v}, \boldsymbol{p}) \geq & r_{j}^{\mathrm{d},(\kappa)}(\boldsymbol{v}, \boldsymbol{p}) \\
\triangleq & a_{j}^{\mathrm{d},(\kappa)}-b_{j}^{\mathrm{d},(\kappa)}\left(\frac{\left(\bar{\lambda}_{j}\left(v_{j}^{(\kappa)}\right)\right)^{2}}{2 \bar{\lambda}_{j}\left(v_{j}^{(\kappa)}\right) \bar{\lambda}_{j}\left(\boldsymbol{v}_{j}\right)-\left(\bar{\lambda}_{j}\left(v_{j}^{(\kappa)}\right)\right)^{2}}\right. \\
& \left.+\frac{\psi_{j}^{\mathrm{d}}(\boldsymbol{v}, \boldsymbol{p})}{\psi_{j}^{\mathrm{d}}\left(v^{(\kappa)}, p^{(\kappa)}\right)}\right)
\end{aligned}
$$

over the trust region

$$
2 \bar{\lambda}_{j}\left(v_{j}^{(\kappa)}\right) \bar{\lambda}_{j}\left(\boldsymbol{v}_{j}\right)-\left(\bar{\lambda}_{j}\left(v_{j}^{(\kappa)}\right)\right)^{2}>0, \quad j=1, \ldots, d,
$$

where $0<a_{j}^{\mathrm{d},(\kappa)} \triangleq r_{j}^{\mathrm{d}}\left(v^{(\kappa)}, p^{(\kappa)}\right)+2 b_{j}^{\mathrm{d},(\kappa)}$, and $0<b_{j}^{\mathrm{d},(\kappa)} \triangleq$ $\frac{\left(\bar{\lambda}_{j}\left(v_{j}^{(\kappa)}\right)\right)^{2}}{\psi_{j}^{\mathrm{d}}\left(v^{(\kappa)}, p^{(\kappa)}\right)+\left(\bar{\lambda}_{j}\left(v_{j}^{(\kappa)}\right)\right)^{2}}$.

Analogously, applying the inequality (73) in the appendix for $\boldsymbol{x}=\boldsymbol{p}_{i}\left|R_{\mathrm{bs}}(i, i)\right|, \boldsymbol{y}=\psi_{\mathrm{bs}}(\boldsymbol{v})$ and $\bar{x}=p_{i}^{(\kappa)}\left|R_{\mathrm{bs}}(i, i)\right|$, $\bar{y}=\psi_{\text {bs }}\left(v^{(\kappa)}\right)$ yields

$$
\begin{aligned}
r_{i}^{\mathrm{u}}\left(\boldsymbol{v}, \boldsymbol{p}_{i}\right) & \geq r_{i}^{\mathrm{u},(\kappa)}\left(\boldsymbol{v}, \boldsymbol{p}_{i}\right) \\
& \triangleq a_{i}^{\mathrm{u},(\kappa)}-b_{i}^{\mathrm{u},(\kappa)}\left(\frac{\left(p_{i}^{(\kappa)}\right)^{2}}{2 p_{i}^{(\kappa)} \boldsymbol{p}_{i}-\left(p_{i}^{(\kappa)}\right)^{2}}+\frac{\psi_{\mathrm{bs}}(\boldsymbol{v})}{\psi_{\mathrm{bs}}\left(v^{(\kappa)}\right)}\right),
\end{aligned}
$$

over the trust region

$$
2 p_{i}^{(\kappa)} \boldsymbol{p}_{i}-\left(p_{i}^{(\kappa)}\right)^{2}>0, \quad i=1, \ldots, u,
$$

where $0<a_{i}^{\mathrm{u},(\kappa)}=r_{i}^{\mathrm{u}}\left(v^{(\kappa)}, p^{(\kappa)}\right)+2 b_{i}^{\mathrm{u},(\kappa)}$ and $b_{i}^{\mathrm{u},(\kappa)}=$ $\frac{\left(p_{i}^{(\kappa)}\left|R_{\mathrm{bs}}(i, i)\right|\right)^{2}}{\psi_{\mathrm{bs}}\left(v^{(\kappa)}\right)+\left(p_{i}^{(\kappa)}\left|R_{\mathrm{bs}}(i, i)\right|\right)^{2}}$.

At the $\kappa$ th iteration we solve the following convex problem to generate the next iterative point $\left(v^{(\kappa+1)}, p^{(\kappa+1)}\right)$ :

$$
\begin{array}{r}
\max _{\boldsymbol{v}, \boldsymbol{p}} L^{(\kappa)}(\boldsymbol{v}, \boldsymbol{p}) \triangleq \sum_{j=1}^{d} r_{j}^{\mathrm{d},(\kappa)}(\boldsymbol{v}, \boldsymbol{p})+\sum_{i=1}^{u} r_{i}^{\mathrm{u},(\kappa)}\left(\boldsymbol{v}, \boldsymbol{p}_{i}\right) \\
-f\left(v^{(\kappa)}, p^{(\kappa)}\right) P^{t o t}(\boldsymbol{v}, \boldsymbol{p})
\end{array}
$$

s.t. $(10 b),(10 c),(11),(12),(16),(18)$.

Note that $L^{(\kappa)}\left(v^{(\kappa)}, p^{(\kappa)}\right)=0$ so $L^{(\kappa)}\left(v^{(\kappa+1)}, p^{(\kappa+1)}\right)>0$ because $\left(v^{(\kappa+1)}, p^{(\kappa+1)}\right)$ is the optimal solution of (19). This means

$$
\begin{aligned}
& \sum_{j=1}^{d} r_{j}^{\mathrm{d},(\kappa)}\left(v^{(\kappa+1)}, p^{(\kappa+1)}\right)+\sum_{i=1}^{u} r_{i}^{\mathrm{u},(\kappa)}\left(v^{(\kappa+1)}, p_{i}^{(\kappa+1)}\right) \\
& -\Phi\left(v^{(\kappa)}, p^{(\kappa)}\right) P^{t o t}\left(v^{(\kappa+1)}, p^{(\kappa+1)}\right)>0 \\
& \sum_{j=1}^{d} r_{j}^{\mathrm{d}}\left(v^{(\kappa+1)}, p^{(\kappa+1)}\right)+\sum_{i=1}^{u} r_{i}^{\mathrm{u}}\left(v^{(\kappa+1)}, p_{i}^{(\kappa+1)}\right) \\
\Leftrightarrow \quad & P^{\operatorname{tot}\left(v^{(\kappa+1)}, p^{(\kappa+1)}\right)} \\
\Leftrightarrow \quad & \Phi\left(v^{(\kappa+1)}, p^{(\kappa+1)}\right)>\Phi\left(v^{(\kappa)}, p^{(\kappa)}\right),
\end{aligned}
$$

i.e. $\left(p^{(\kappa+1)}, v^{(\kappa+1)}\right)$ is a better feasible point than $\left(p^{(\kappa)}, v^{(\kappa)}\right)$ for the nonconvex optimization problem (13). Then it is easy to show that the sequence $\left\{\left(p^{(\kappa)}, v^{(\kappa)}\right)\right\}$ converges at least to a locally optimal solution of (13)/(10) [34]. It has been shown e.g. in [20] that such a locally optimal solution often turns out to be the global one. A pseudo-code of the proposed iterative process is given by Algorithm 1.

\begin{tabular}{l}
\hline Algorithm 1 Full-duplex EE Optimization Algorithm \\
\hline 1: Initialization: Set $\kappa=0$. Solve the follow- \\
ing convex problem for initial point $\left(v^{(0)}, p^{(0)}\right)$ : \\
$\min _{\boldsymbol{v}, \boldsymbol{p}} \sum_{j=1}^{d}\left\|\boldsymbol{v}_{j}\right\|^{2}+\sum_{i=1}^{u} \boldsymbol{p}_{i}^{2}$ subject to $(10 \mathrm{~b}),(10 \mathrm{c})$, \\
(11), (12).
\end{tabular}

2: Repeat until convergence: Solve the convex problem (19) to generate the next feasible point $\left(v^{(\kappa+1)}, p^{(\kappa+1)}\right)$ for (10). Set $\kappa:=\kappa+1$.

3: Output $\left(v^{(\kappa)}, p^{(\kappa)}\right)$ as the optimal solution of (10).

Although Algorithm 1 provides means for efficient computation of the FD-based STR's EE optimization problem (10), one can see the following inherent drawbacks of FD-based STR preventing it from achieving high EE or providing high QoS:

- The DL throughput defined by (2) is UL interferencelimited: the UL interference in (1) can be strong and uncontrolled whenever there is a UL near to a DL.

- The UL throughput defined by (8) is SI-limited: the SI in (3), which is proportional to the BS transmit power as (5) shows, is very strong due to the co-location of BS transmit and receive antennas. It is technologically impossible to suppress it to the background noise level even for microcells with much weaker transmit signals.

- Only $n_{1}$ antennas are used for signal transmission and $n_{2}$ antennas are used for signal reception, restricting the number of served downlinks and uplinks up to $n_{1}$ and $n_{2}$ respectively.

The next two sections provide quite different STRs to resolve these drawbacks.

\section{TIME-FRACTION-WISE STR}

We now propose the first alternative approach, which not only resolves all the above issues of (micro) FD-based STR but also works for macrocells. Still within a single time slot, the $\mathrm{BS}$ uses $n_{1}$ antennas to transmit signal during a fraction $0<\tau<1$ of the time-slot to serve DLs as illustrated by Fig. 2(a) and then users the remaining $n_{2}$ antennas in the remaining fraction $1-\tau$ to receive signal from ULs, who are kept silent during the first $\tau$ fraction, as illustrated by Fig. 2(b). Inter-link interference and SI are thus completely avoided.

Moreover, as illustrated by Fig. 3, all $n$ antennas can be used to transmit signal during a fraction $0<\tau<1$ of the time-slot to serve DLs, which are then switched in a fraction $\epsilon$ of the time-slot to receive signal from ULs in the remaining fraction $1-\epsilon-\tau$. Thus, the full number of antennas is utilized for transmission and reception with is no inter-link interference and SI. We refer the first option as TF while the second option as e-TF to distinguish them.

Now, let $\widetilde{\boldsymbol{v}}_{j} \in \mathbb{C}^{n_{t} \times d_{1}}$ be the beamforming vector for $\mathrm{DL}_{j}$, so $n_{t}=n_{1}$ for TF while $n_{t}=n$ for e-TF. For computational tractability, the power allocated to $s_{i}^{\mathrm{u}}$ is defined by $1 / \boldsymbol{p}_{i}$ instead of $\boldsymbol{p}_{i}^{2}$ in the previous section. 


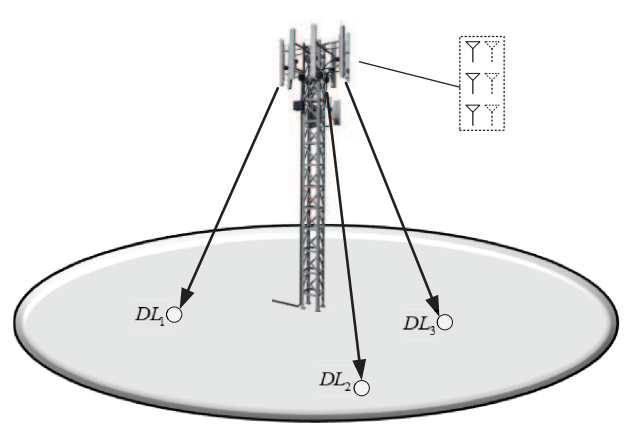

(a)

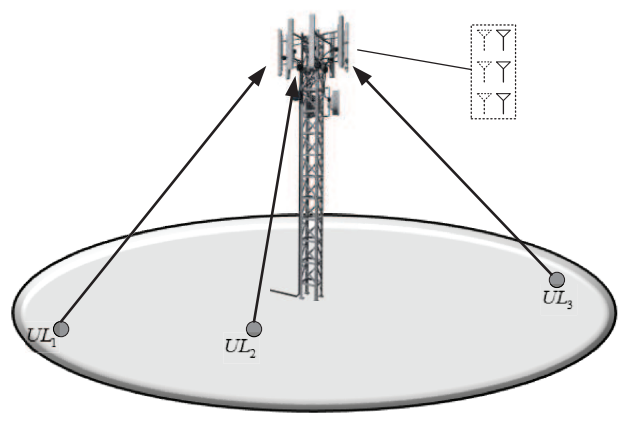

(b)

Fig. 2: TF/BF STR system: (a) Signal transmit during a fraction $\tau$ of time slot/bandwdith; (b) Signal reception during the remaining fraction $1-\tau$ of time slot/bandwidth

The received signal at DLU $j$ is now:

$$
\tilde{y}_{j} \triangleq \underbrace{\tilde{h}_{j, \mathrm{bs}}^{H} \widetilde{\boldsymbol{v}}_{j} s_{j}^{\mathrm{d}}}_{\text {desired signal }}+\underbrace{\sum_{\ell \in \mathcal{D} \backslash\{j\}} \tilde{h}_{j, \mathrm{bs}}^{H} \widetilde{\boldsymbol{v}}_{\ell} s_{\ell}^{\mathrm{d}}}_{\text {DL interference }}+n_{j}, j=1, \ldots, d,
$$

where $\tilde{h}_{j, \text { bs }} \in \mathbb{C}^{n_{t}}$ is the downlink channel from the BS to $\mathrm{DL}_{j}$.

For $\widetilde{\boldsymbol{v}} \triangleq\left\{\widetilde{\boldsymbol{v}}_{j}, j=1, \ldots, d\right\}$, the throughput at $\mathrm{DL}_{j}$ is $\boldsymbol{\tau} \rho_{j}^{\mathrm{d}}(\widetilde{\boldsymbol{v}})$ with

$$
\rho_{j}^{\mathrm{d}}(\widetilde{\boldsymbol{v}}) \triangleq \ln \left(1+\frac{\left|\widetilde{\lambda}_{j}\left(\widetilde{\boldsymbol{v}}_{j}\right)\right|^{2}}{\phi_{j}^{\mathrm{d}}(\widetilde{\boldsymbol{v}})}\right),
$$

where $\widetilde{\lambda}_{j}\left(\widetilde{\boldsymbol{v}}_{j}\right) \triangleq \tilde{h}_{j, \mathrm{bs}}^{H} \widetilde{\boldsymbol{v}}_{j}$ and $\phi_{j}^{\mathrm{d}}(\widetilde{\boldsymbol{v}}) \triangleq \sum_{\ell \in \mathcal{D} \backslash\{j\}}\left|\tilde{h}_{j, \mathrm{bs}}^{H} \widetilde{\boldsymbol{v}}_{\ell}\right|^{2}+\sigma_{\mathrm{d}}^{2}$.

The received signal at the $\mathrm{BS}$ is now

$$
\widetilde{y}_{\mathrm{bs}} \triangleq \sum_{i=1}^{u} \tilde{h}_{\mathrm{bs}, i} s_{i}^{\mathrm{u}} / \sqrt{\boldsymbol{p}_{i}}+\widetilde{n}_{\mathrm{bs}},
$$

where $\tilde{h}_{\mathrm{bs}, i} \in \mathbb{C}^{n_{r}}$ is the uplink channel from $\mathrm{UL}_{i}$ to the BS ( $n_{r}=n_{2}$ for TF and $n_{r}=n$ for e-TF), and $\widetilde{n}_{\mathrm{bs}}$ is the additive white Gaussian noise with variance $\sigma_{\mathrm{bs}}^{2} I_{n_{r}}$. Again, making QR decomposition

$$
\left[\begin{array}{llll}
\tilde{h}_{\mathrm{bs}, 1} & \tilde{h}_{\mathrm{bs}, 2} & \ldots & \tilde{h}_{\mathrm{bs}, u}
\end{array}\right]=\tilde{Q}_{\mathrm{bs}} \tilde{R}_{\mathrm{bs}}
$$

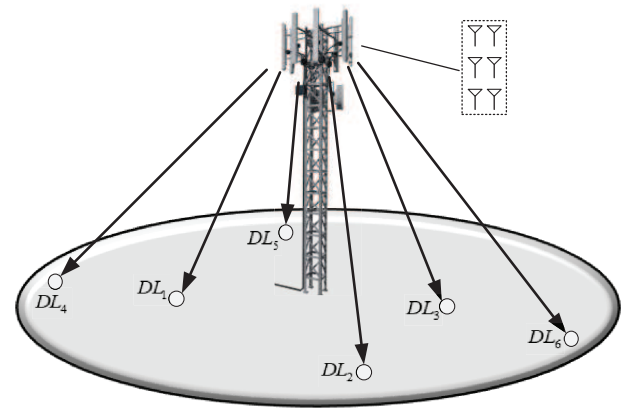

(a)

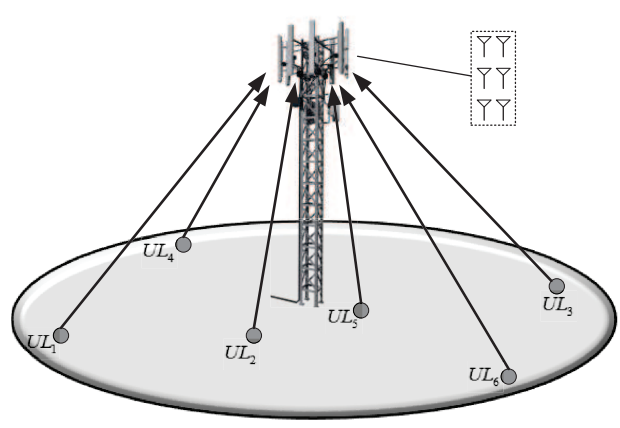

(b)

Fig. 3: e-TF STR system: (a) Signal transmit during a fraction $\tau$ of time slot by all antennas; (b) Signal reception during the remaining fraction $\eta-\tau$ of time slot by the same antennas

with an unitary matrix $\tilde{Q}_{\mathrm{bs}}$ of size $n_{r} \times n_{r}$ and an upper rectangular matrix $\tilde{R}_{\text {bs }}$ of size $n_{r} \times u$, the uplink throughput of $s_{i}^{\mathrm{u}}$ at the BS is $(\eta-\boldsymbol{\tau}) \rho_{i}^{\mathrm{u}}\left(\boldsymbol{p}_{i}\right)$ with $\eta=1$ for TF and $\eta=1-\epsilon$ for $\mathrm{e}-\mathrm{FT}$, and

$$
\rho_{i}^{\mathrm{u}}\left(\boldsymbol{p}_{i}\right) \triangleq \ln \left(1+\left|\tilde{R}_{\mathrm{bs}}(i, i)\right|^{2} / \sigma_{\mathrm{bs}}^{2} \boldsymbol{p}_{i}\right) .
$$

For $\boldsymbol{p} \triangleq\left\{\boldsymbol{p}_{i}, i=1, \ldots, u\right\}$, instead of (9), the consumed power is modelled as

$$
P^{t o t}(\widetilde{\boldsymbol{v}}, \boldsymbol{p}, \boldsymbol{\tau})=\zeta P_{i}^{t}(\widetilde{\boldsymbol{v}}, \boldsymbol{p}, \boldsymbol{\tau})+P_{B S}+P_{U E},
$$

where $P_{i}^{t}(\widetilde{\boldsymbol{v}}, \boldsymbol{p}, \boldsymbol{\tau}) \triangleq \boldsymbol{\tau} \sum_{j=1}^{d}\left\|\widetilde{\boldsymbol{v}}_{j}\right\|^{2}+(\eta-\boldsymbol{\tau}) \sum_{i=1}^{u} 1 / \boldsymbol{p}_{i}$ is the total transmit power of the BS and UEs, which is no longer a convex function. The time-fraction-wise (TF-wise) STR's EE 
optimization problem is now formulated as

$$
\begin{array}{r}
\max _{0<\boldsymbol{\tau}<1, \widetilde{\boldsymbol{v}}, \boldsymbol{p}} \frac{\boldsymbol{\tau} \sum_{j=1}^{d} \rho_{j}^{\mathrm{d}}(\widetilde{\boldsymbol{v}})+(\eta-\boldsymbol{\tau}) \sum_{i=1}^{u} \rho_{i}^{\mathrm{u}}\left(\boldsymbol{p}_{i}\right)}{P^{t o t}(\widetilde{\boldsymbol{v}}, \boldsymbol{p}, \boldsymbol{\tau})} \\
\boldsymbol{\tau} \sum_{j=1}^{d}\left\|\widetilde{\boldsymbol{v}}_{j}\right\|^{2} \leq P_{\mathrm{BS}}^{\max }, \\
(\eta-\boldsymbol{\tau}) / \boldsymbol{p}_{i} \leq P_{\mathrm{UE}}^{\max }, \quad i=1, \ldots, u, \\
\boldsymbol{\tau} \rho_{j}^{\mathrm{d}}(\widetilde{\boldsymbol{v}}) \geq r_{\mathrm{d}}, \quad j=1, \ldots, d, \\
(\eta-\boldsymbol{\tau}) \rho_{i}^{\mathrm{u}}\left(\boldsymbol{p}_{i}\right) \geq r_{\mathrm{u}}, \quad i=1, \ldots, u, \\
\left\|\widetilde{\boldsymbol{v}}_{j}\right\|^{2} \leq P_{\mathrm{D}}^{\max }, \quad j=1, \ldots, d, \\
1 / P_{\mathrm{U}}^{\max } \leq \boldsymbol{p}_{i}, \quad i=1, \ldots, u,
\end{array}
$$

where (26f) and (26g) caps the physical limit of transmit power rates for the BS and ULs. Compared to the EE optimization problem (13) for the FD-based STR, the problem (26) is more computationally difficult because not only the objective function in (26) exhibits a more complex structure but all constraints (26b)-(26e) are no longer computationally tractable due to the presence of the time-fraction variable $\tau$. By introducing the variable $\boldsymbol{\theta}=\left(\boldsymbol{\theta}_{1}, \boldsymbol{\theta}_{2}\right)$, which satisfies the convex constraint

$$
\boldsymbol{\tau} \geq 1 / \boldsymbol{\theta}_{1}>0, \quad \eta-\boldsymbol{\tau} \geq 1 / \boldsymbol{\theta}_{2}>0,
$$

the problem (26) is equivalently expressed by

$$
\begin{array}{r}
\max _{\boldsymbol{\tau}, \boldsymbol{\theta}, \widetilde{\boldsymbol{v}}, \boldsymbol{p}} \tilde{\Phi}(\widetilde{\boldsymbol{v}}, \boldsymbol{p}, \boldsymbol{\theta}) \triangleq \frac{\sum_{j=1}^{d} \rho_{j}^{\mathrm{d}}(\widetilde{\boldsymbol{v}}) / \boldsymbol{\theta}_{1}+\sum_{i=1}^{u} \rho_{i}^{\mathrm{u}}\left(\boldsymbol{p}_{i}\right) / \boldsymbol{\theta}_{2}}{\tilde{P} \tilde{P}^{t o t}(\widetilde{\boldsymbol{v}}, \boldsymbol{x}, \boldsymbol{\theta})} \\
\text { s.t. }(26 f),(26 g),(27), \\
\sum_{j=1}^{d}\left\|\widetilde{\boldsymbol{v}}_{j}\right\|^{2} \leq P_{\mathrm{BS}}^{\max } \boldsymbol{\theta}_{1}, \\
1 / \boldsymbol{p}_{i} \leq P_{\mathrm{UE}}^{\max } \boldsymbol{\theta}_{2}, \quad i=1, \ldots, u, \\
\rho_{j}^{\mathrm{d}}(\widetilde{\boldsymbol{v}}) \geq \boldsymbol{\theta}_{1} r_{\mathrm{d}}, \quad j=1, \ldots, d, \\
\rho_{i}^{\mathrm{u}}\left(\boldsymbol{p}_{i}\right) \geq \boldsymbol{\theta}_{2} r_{\mathrm{u}}, \quad i=1, \ldots, u,
\end{array}
$$

for

$\tilde{P}^{t o t}(\widetilde{\boldsymbol{v}}, \boldsymbol{x}, \boldsymbol{\theta}) \triangleq \zeta\left(\sum_{j=1}^{d} \frac{\left\|\widetilde{\boldsymbol{v}}_{j}\right\|^{2}}{\boldsymbol{\theta}_{1}}+\sum_{i=1}^{u} \frac{1}{\boldsymbol{x}_{i} \boldsymbol{\theta}_{2}}\right)+P^{B S}+U P^{U E}$,

which becomes a convex function. Note that the constraints and (26f), (26g), (27), (28b), and (28c) in (28) are now convex, while the constraints (28d) and (28e) are nonconvex. Let $\left(\tilde{v}^{(\kappa)}, p^{(\kappa)}, \theta^{(\kappa)}\right)$ be a feasible point for (28) found from the $(\kappa-1)$ th iteration.

Applying the inequalities (73) and (72) in the appendix for $\boldsymbol{x}=\widetilde{\lambda}_{j}\left(\widetilde{\boldsymbol{v}}_{j}\right), \boldsymbol{y}=\phi_{j}^{\mathrm{d}}(\widetilde{\boldsymbol{v}}), \boldsymbol{t}=\boldsymbol{\theta}_{1}$ and $\bar{x}=\widetilde{\lambda}_{j}\left(\tilde{v}_{j}^{(\kappa)}\right), \bar{y}=$ $\phi_{j}^{\mathrm{d}}\left(\tilde{v}^{(\kappa)}\right), \bar{t}=\theta_{1}^{(\kappa)}$ yields

$$
\rho_{j}^{\mathrm{d}}(\widetilde{\boldsymbol{v}}) \geq \rho^{\mathrm{d},(\kappa)}(\widetilde{\boldsymbol{v}}) \triangleq
$$

$$
\begin{array}{r}
\tilde{a}_{j}^{\mathrm{d},(\kappa)}-\tilde{b}_{j}^{\mathrm{d},(\kappa)}\left(\frac{\left|\widetilde{\lambda}_{j}\left(\tilde{v}_{j}^{(\kappa)}\right)\right|^{2}}{2 \Re\left\{\left(\widetilde{\lambda}_{j}\left(\tilde{v}_{j}^{(\kappa)}\right)\right) * \widetilde{\lambda}_{j}\left(\widetilde{\boldsymbol{v}}_{j}\right)\right\}-}-\left|\widetilde{\lambda}_{j}\left(\tilde{v}_{j}^{(\kappa)}\right)\right|^{2}\right. \\
\left.+\frac{\phi_{j}^{\mathrm{d}}(\widetilde{\boldsymbol{v}})}{\phi_{j}^{\mathrm{d}}\left(\tilde{v}^{(\kappa)}\right)}\right)
\end{array}
$$

and

$$
\begin{array}{r}
\frac{\rho_{j}^{\mathrm{d}}(\widetilde{\boldsymbol{v}})}{\boldsymbol{\theta}_{1}} \geq \tilde{f}_{j}^{\mathrm{d},(\kappa)}\left(\widetilde{\boldsymbol{v}}, \boldsymbol{\theta}_{1}\right) \triangleq \\
\tilde{\tilde{a}}_{j}^{\mathrm{d},(\kappa)}-\tilde{\tilde{b}}_{j}^{\mathrm{d},(\kappa)}\left(\frac{\left|\widetilde{\lambda}_{j}\left(\tilde{v}_{j}^{(\kappa)}\right)\right|^{2}}{2 \Re\left\{\left(\widetilde{\lambda}_{j}\left(\tilde{v}_{j}^{(\kappa)}\right)\right) * \widetilde{\lambda}_{j}\left(\widetilde{\boldsymbol{v}}_{j}\right)\right\}-\left|\widetilde{\lambda}_{j}\left(\tilde{v}_{j}^{(\kappa)}\right)\right|^{2}}\right. \\
\left.+\frac{\phi_{j}^{\mathrm{d}}(\widetilde{\boldsymbol{v}})}{\phi_{j}^{\mathrm{d}}\left(\tilde{v}^{(\kappa)}\right)}\right)-\tilde{\tilde{c}}_{j}^{\mathrm{d},(\kappa)} \boldsymbol{\theta}_{1}
\end{array}
$$

over the trust region

$$
2 \Re\left\{\left(\widetilde{\lambda}_{j}\left(\tilde{v}_{j}^{(\kappa)}\right)\right)^{*} \widetilde{\lambda}_{j}\left(\widetilde{\boldsymbol{v}}_{j}\right)\right\}-\left|\widetilde{\lambda}_{j}\left(\tilde{v}_{j}^{(\kappa)}\right)\right|^{2}>0, \quad j=1, \ldots, d,
$$

where $0<\tilde{a}_{j}^{\mathrm{d},(\kappa)}=\rho_{j}^{\mathrm{d}}\left(\tilde{v}^{(\kappa)}\right)+2 \tilde{b}_{j}^{\mathrm{d},(\kappa)}, 0<\tilde{b}_{j}^{\mathrm{d},(\kappa)}=$ $\frac{\left|\widetilde{\lambda}_{j}\left(\tilde{v}_{j}^{(\kappa)}\right)\right|^{2}}{\phi_{j}^{\mathrm{d}}\left(\tilde{v}^{(\kappa)}\right)+\left(\left.\widetilde{\lambda}_{j}\left(\tilde{v}_{j}^{(\kappa)}\right)\right|^{2}\right.}$, and $0<\tilde{\tilde{a}}_{j}^{\mathrm{d},(\kappa)}=2 \frac{\rho_{j}^{\mathrm{d}}\left(\tilde{v}^{(\kappa)}\right)}{\theta_{1}^{(\kappa)}}+2 \tilde{\tilde{b}}_{j}^{\mathrm{d},(\kappa)}$, $0<\tilde{\tilde{b}}_{j}^{\mathrm{d},(\kappa)}=\frac{\left|\widetilde{\lambda}_{j}\left(\tilde{v}_{j}^{(\kappa)}\right)\right|^{2}}{\left(\phi_{j}^{\mathrm{d}}\left(\tilde{v}^{(\kappa)}\right)+\left.\tilde{\lambda}_{j}\left(\tilde{v}_{j}^{(\kappa)}\right)\right|^{2}\right) \theta_{1}^{(\kappa)}}, 0<\tilde{\tilde{c}}_{j}^{\mathrm{d},(\kappa)}=\frac{\rho_{j}^{\mathrm{d}}\left(\tilde{v}^{(\kappa)}\right)}{\left(\theta_{1}^{(\kappa)}\right)^{2}}$. The nonconvex constraint (28d) is innerly approximated by the following convex constraint

$$
\rho^{\mathrm{d},(\kappa)}(\widetilde{\boldsymbol{v}}) \geq \boldsymbol{\theta}_{1} r_{\mathrm{d}}, \quad j=1, \ldots, d,
$$

i.e. any feasible point for (33) is also feasible for (28d).

Applying the inequalities (65) and (64) in the appendix for $\boldsymbol{x}=\boldsymbol{p}_{i} \sigma_{\mathrm{bs}}^{2} /\left|\tilde{R}_{\mathrm{bs}}(i, i)\right|^{2}, \boldsymbol{t}=\boldsymbol{\theta}_{2}$, and $\bar{x}=p_{i}^{(\kappa)} \sigma_{\text {bs }}^{2} /\left|\tilde{R}_{\mathrm{bs}}(i, i)\right|^{2}$, $\bar{t}=\theta_{2}^{(\kappa)}$ yields

$$
\rho_{i}^{\mathrm{u}}\left(\boldsymbol{p}_{i}\right) \geq \rho_{i}^{\mathrm{u},(\kappa)}\left(\boldsymbol{p}_{i}\right) \triangleq a_{i}^{\mathrm{u},(\kappa)}-b_{i}^{\mathrm{u},(\kappa)} \frac{\boldsymbol{p}_{i}}{p_{i}^{(\kappa)}}
$$

and

$$
\frac{\rho_{i}^{\mathrm{u}}\left(\boldsymbol{p}_{i}\right)}{\boldsymbol{\theta}_{2}} \geq \tilde{f}_{i}^{\mathrm{u},(\kappa)}\left(\boldsymbol{p}_{i}, \boldsymbol{\theta}_{2}\right) \triangleq \tilde{a}_{i}^{\mathrm{u},(\kappa)}-\tilde{b}_{i}^{\mathrm{u},(\kappa)} \frac{\boldsymbol{p}_{i}}{p_{i}^{(\kappa)}}-\tilde{c}_{i}^{\mathrm{u},(\kappa)} \boldsymbol{\theta}_{2},
$$

where $0<a_{i}^{\mathrm{u},(\kappa)}=\rho_{i}^{\mathrm{u}}\left(p_{i}^{(\kappa)}\right)+b_{i}^{\mathrm{u},(\kappa)}, b_{i}^{\mathrm{u},(\kappa)}=$ $\frac{\left|\tilde{R}_{\mathrm{bs}}(i, i)\right|^{2}}{p_{i}^{(\kappa)} \sigma_{\mathrm{bs}}^{2}+\left|\tilde{R}_{\mathrm{bs}}(i, i)\right|^{2}}$, and $0<\tilde{a}_{i}^{\mathrm{u},(\kappa)}=2 \frac{\rho_{i}^{\mathrm{u}}\left(p_{i}^{(\kappa)}\right)}{\theta_{2}^{(\kappa)}}+\tilde{b}_{i}^{\mathrm{u},(\kappa)}, \tilde{b}_{i}^{\mathrm{u},(\kappa)}=$ $\frac{\left|\tilde{R}_{\mathrm{bs}}(i, i)\right|^{2}}{\left(p_{i}^{(\kappa)} \sigma_{\mathrm{bs}}^{2}+\left|\tilde{R}_{\mathrm{bs}}(i, i)\right|^{2}\right) \theta_{2}^{(\kappa)}}, \tilde{c}_{i}^{\mathrm{u},(\kappa)}=\frac{\rho_{i}^{u}\left(p_{i}^{(\kappa)}\right)}{\left(\theta_{2}^{(\kappa)}\right)^{2}}$. The nonconvex constraint $(28 \mathrm{e})$ is innerly approximated by the following convex constraint

$$
\rho_{i}^{\mathrm{u},(\kappa)}\left(\boldsymbol{p}_{i}\right) \geq \boldsymbol{\theta}_{2} r_{\mathrm{u}}, \quad i=1, \ldots, u .
$$

At the $\kappa$ th iteration we solve the following convex problem to generate the next feasible point $\left(\tilde{v}^{(\kappa+1)}, p^{(\kappa+1)}, \theta^{(\kappa+1)}\right)$ :

$$
\begin{aligned}
& \max _{\widetilde{\boldsymbol{v}}, \boldsymbol{p}, \boldsymbol{\theta}, \boldsymbol{\tau}} \tilde{L}^{(\kappa)}(\widetilde{\boldsymbol{v}}, \boldsymbol{p}, \boldsymbol{\theta}) \triangleq \sum_{j=1}^{d} \tilde{f}_{j}^{\mathrm{d},(\kappa)}\left(\widetilde{\boldsymbol{v}}, \boldsymbol{\theta}_{1}\right) \\
&+\sum_{i=1}^{u} \tilde{f}_{i}^{\mathrm{u},(\kappa)}\left(\boldsymbol{x}_{i}, \boldsymbol{\theta}_{2}\right)-\tilde{\Phi}\left(\tilde{v}^{(\kappa)}, x^{(\kappa)}, \theta^{(\kappa)}\right) \tilde{P}^{t o t}(\widetilde{\boldsymbol{v}}, \boldsymbol{p}, \boldsymbol{\theta}) \\
& \text { s.t. } \quad(26 f),(26 g),(27),(28 b),(32),(33),(36) .
\end{aligned}
$$


Note that any feasible point for the convex problem (37) is also feasible for the nonconvex problem (28). Also, $\tilde{L}^{(\kappa)}\left(\tilde{v}^{(\kappa)}, p^{(\kappa)}, \theta^{(\kappa)}\right)=0$ so $\tilde{L}^{(\kappa)}\left(\tilde{v}^{(\kappa+1)}, p^{(\kappa+1)}, \theta^{(\kappa+1)}\right)>0$ at the optimal solution of (37) as far as $\left(\tilde{v}^{(\kappa)}, p^{(\kappa)}, \theta^{(\kappa)}\right) \neq$ $\left(\tilde{v}^{(\kappa+1)}, p^{(\kappa+1)}, \theta^{(\kappa+1)}\right)$. Like (20),we then have

$$
\tilde{\Phi}\left(\tilde{v}^{(\kappa+1)}, p^{(\kappa+1)}, \theta^{(\kappa+1)}\right)>\tilde{\Phi}\left(\tilde{v}^{(\kappa)}, p^{(\kappa)}, \theta^{(\kappa)}\right),
$$

i.e. $\left(\tilde{v}^{(\kappa+1)}, p^{(\kappa+1)}, \theta^{(\kappa+1)}\right)$ is a better feasible for (28) than $\left(\tilde{v}^{(\kappa)}, p^{(\kappa)}, \theta^{(\kappa)}\right)$. As such, the sequence $\left\{\left(\tilde{v}^{(\kappa)}, p^{(\kappa)}, \theta^{(\kappa)}\right)\right\}$ converges at least to a locally optimal solution of (28).

It is important to locate a feasible point $\left(\tilde{v}^{(0)}, p^{(0)}, \theta^{(0)}\right)$ for (28) for initialization as follows: under fixed $\tau^{(0)}$ and $\left(\theta_{1}^{(0)}, \theta_{2}^{(0)}\right)=\left(1 / \tau^{(0)}, 1 /\left(\eta-\tau^{(0)}\right)\right)$, by changing variable if necessary one can replace $\left|\tilde{\lambda}_{j}\left(\tilde{\boldsymbol{v}}_{j}\right)\right|^{2}$ by $\left(\bar{\lambda}_{j}\left(\tilde{\boldsymbol{v}}_{j}\right)\right)^{2}$ for $\bar{\lambda}_{j}\left(\tilde{\boldsymbol{v}}_{j}\right)=\Re\left\{\tilde{\lambda}_{j}\left(\tilde{\boldsymbol{v}}_{j}\right)\right\}$ in (22), so

$$
\rho_{j}^{\mathrm{d}}(\tilde{\boldsymbol{v}})=\ln \left(1+\frac{\left(\bar{\lambda}_{j}\left(\tilde{\boldsymbol{v}}_{j}\right)\right)^{2}}{\phi_{j}(\tilde{\boldsymbol{v}})}\right),
$$

and (26d) is equivalent to the SOC constraint

$$
\begin{aligned}
\bar{\lambda}_{j}\left(\tilde{\boldsymbol{v}}_{j}\right) \geq & \sqrt{e^{r_{\mathrm{d}} / \tau^{(0)}}-1} \sqrt{\phi_{j}(\tilde{\boldsymbol{v}})} \\
= & \sqrt{e^{r_{\mathrm{d}} / \tau^{(0)}}-1}\left\|\left(\tilde{h}_{j, \mathrm{bs}}^{H} \tilde{\boldsymbol{v}}_{\ell}\right)_{\ell \in \mathcal{D} \backslash\{j\}}\right\|_{\mathrm{d}}, \\
& j=1, \ldots, d,
\end{aligned}
$$

while (26e) is also equivalent to the following linear constraint

$$
\left|\tilde{R}_{\mathrm{bs}}(i, i)\right| \geq \boldsymbol{p}_{i} \sqrt{e^{r_{\mathrm{u}} /\left(\eta-\tau^{(0)}\right)}-1} \sigma_{\mathrm{bs}}, \quad i=1, \ldots, u .
$$

We then solve the following convex problem to generate $\left(\tilde{v}^{(0)}, p^{(0)}\right)$

$$
\begin{array}{r}
\min _{\widetilde{\boldsymbol{v}}, \boldsymbol{p}} \sum_{j=1}^{d}\left\|\tilde{\boldsymbol{v}}_{j}\right\|^{2}+\sum_{i=1}^{u} \frac{1}{\boldsymbol{p}_{i}} \quad \text { s.t. } \quad(39),(40), \\
\sum_{j=1}^{d}\left\|\widetilde{\boldsymbol{v}}_{j}\right\|^{2} \leq \frac{P_{\mathrm{BS}}^{\max }}{\tau^{(0)}},\left\|\widetilde{\boldsymbol{v}}_{j}\right\|^{2} \leq P_{\mathrm{D}}^{\max }, \quad j=1, \ldots, d, \\
1 \leq \frac{P_{\mathrm{UE}}^{\max }}{\eta-\tau^{(0)}} \boldsymbol{p}_{i}, 1 / P_{\mathrm{U}}^{\max } \leq \boldsymbol{p}_{i}, \quad i=1, \ldots, u .
\end{array}
$$

Algorithm 2 summarizes our proposed computational procedure for solving (28).

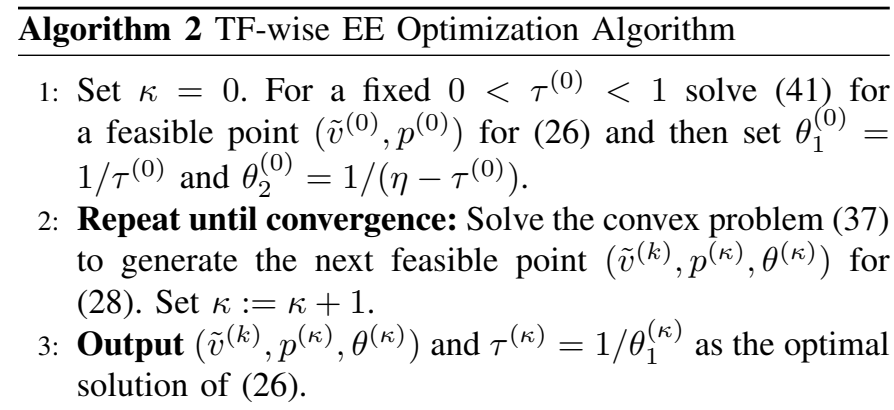

\section{BANDWIDTH-FRACTION-WISE STR}

Instead of time-fraction allocation for serving uplinks and downlinks, we now consider a bandwidth allocation for their service, i.e. a portion $0<\tau<1$ of the whole normalized bandwidth is allocated to the downlink service, the portion $\epsilon$ for guarding and the remaining complementary portion $(\eta-\tau)$ with $\eta=1-\epsilon$ is allocated to the uplink service. Unlike two approaches presented in the previous section, this bandwidth-fraction-wise STR can be practically implemented only when the portion $\tau$ is determined and fixed beforehand. In simulation, we also provide numerical for $\tau=1 / 2$, i.e. a half of the bandwidth is used for serving the uplinks and another half of the bandwidth is used for serving downlinks.

As in (1), let $h_{j, \text { bs }} \in \mathbb{C}^{n_{1}}$ be the channel vector from the BS to $\mathrm{DL}_{j}$ and $\boldsymbol{v}_{j}$ be the beamformer for the information $s_{j}^{\mathrm{d}}$ intended for $\mathrm{DL}_{j}$. The received signal at $\mathrm{DL}_{j}$ is

$$
\hat{y}_{j} \triangleq \underbrace{h_{j, \mathrm{bs}}^{H} \boldsymbol{v}_{j} s_{j}^{\mathrm{d}}}_{\text {desired signal }}+\underbrace{\sum_{\ell \in \mathcal{D} \backslash\{j\}} h_{j, \mathrm{bs}}^{H} \boldsymbol{v}_{\ell} s_{\ell}^{\mathrm{d}}}_{\text {DL interference }}+n_{j}(\boldsymbol{\tau}), j=1, \ldots, d,
$$

where $n_{j}(\boldsymbol{\tau})$ is the additive white circularly symmetric complex Gaussian noise with the variance $\tau \sigma_{\mathrm{d}}^{2}$. Unlike (1), the received signal $y_{j}$ is now free from the UL interference as the BS's broadcast and ULs' transmit are implemented in orthogonal frequency bands.

The throughput at $\mathrm{DL}_{j}$ is $\boldsymbol{\tau} \varphi_{j}^{\mathrm{d}}(\boldsymbol{v}, \boldsymbol{\tau})$ with

$$
\varphi_{j}^{\mathrm{d}}(\boldsymbol{v}, \boldsymbol{\tau}) \triangleq \ln \left(1+\frac{\left|\tilde{\lambda}_{j}\left(\boldsymbol{v}_{j}\right)\right|^{2}}{v_{j}(\boldsymbol{v}, \boldsymbol{\tau})}\right),
$$

where $\tilde{\lambda}_{j}\left(\boldsymbol{v}_{j}\right) \triangleq h_{j, \mathrm{bs}}^{H} \widetilde{\boldsymbol{v}}_{j}$ as in (2), and $v_{j}(\boldsymbol{v}, \boldsymbol{\tau}) \triangleq$ $\sum_{\ell \in \mathcal{D} \backslash\{j\}}\left|h_{j, \mathrm{bs}}^{H} \boldsymbol{v}_{\ell}\right|^{2}+\boldsymbol{\tau} \sigma_{\mathrm{d}}^{2}$.

As in (4), let $h_{\mathrm{bs}, i} \in \mathbb{C}^{n_{2}}$ be the channel vector from $\mathrm{UL}_{i}$ to the $\mathrm{BS}$, and $s_{i}^{\mathrm{u}}$ be the information $\mathrm{UL}_{i}$ intends to send to the BS. The received signal at the BS is

$$
\hat{y}_{\mathrm{bs}} \triangleq \sum_{i=1}^{u} h_{\mathrm{bs}, i} \sqrt{\boldsymbol{p}_{i}} s_{i}^{\mathrm{u}}+n_{\mathrm{bs}}(\boldsymbol{\tau}),
$$

where $\boldsymbol{p}_{i}$ is the $\mathrm{UL}_{i}{ }^{\prime}$ transmit power, and $n_{\mathrm{bs}}(\boldsymbol{\tau})$ is the additive white circularly symmetric Gaussian noise with variance $(\eta-$ $\tau) \sigma_{\text {bs }}^{2} I_{N_{2}}$. The achievable uplink throughput for $s_{i}^{\mathrm{u}}$ at the BS is $(\eta-\boldsymbol{\tau}) \varphi_{i}^{\mathrm{u}}\left(\boldsymbol{p}_{i}, \boldsymbol{\tau}\right)$ with

$$
\varphi_{i}^{\mathrm{u}}(\boldsymbol{p}, \boldsymbol{\tau}) \triangleq \ln \left(1+\frac{\boldsymbol{p}_{i}\left|\tilde{R}_{\mathrm{bs}}(i, i)\right|^{2}}{(\eta-\boldsymbol{\tau}) \sigma_{\mathrm{bs}}^{2}}\right) .
$$

The consumed power $P^{t o t}$ is modelled by the following convex quadratic function

$$
P^{t o t}(\boldsymbol{v}, \boldsymbol{p})=\zeta P_{i}^{t}(\boldsymbol{v}, \boldsymbol{p})+P^{B S}+U P^{U E},
$$

where $P^{t}(\boldsymbol{v}, \boldsymbol{p}) \triangleq \sum_{j=1}^{d}\left\|\boldsymbol{v}_{j}\right\|^{2}+\sum_{i=1}^{u} \boldsymbol{p}_{i}$. 
The bandwidth-fraction-wise (BF-wise) STR's EE optimization problem is formulated as

$$
\begin{aligned}
& \max _{0<\boldsymbol{\tau}<1, \boldsymbol{v}, \boldsymbol{p}} \hat{\Phi}(\boldsymbol{v}, \boldsymbol{p}, \boldsymbol{\tau}) \triangleq \\
& \frac{\boldsymbol{\tau} \sum_{j=1}^{d} \varphi_{j}^{\mathrm{d}}(\boldsymbol{v}, \boldsymbol{\tau})+(\eta-\boldsymbol{\tau}) \sum_{i=1}^{u} \varphi_{i}^{\mathrm{u}}\left(\boldsymbol{p}_{i}, \boldsymbol{\tau}\right)}{P^{t o t}(\boldsymbol{v}, \boldsymbol{p})} \text { s.t. } \\
& \sum_{j=1}^{d}\left\|\boldsymbol{v}_{j}\right\|^{2} \leq P_{\mathrm{BS}}^{\max }, \\
& \boldsymbol{p}_{i} \leq P_{\mathrm{UE}}^{\max }, \quad i=1, \ldots, u, \\
& \boldsymbol{\tau} \varphi_{j}^{\mathrm{d}}(\boldsymbol{v}, \boldsymbol{\tau}) \geq r_{\mathrm{d}}, \quad j=1, \ldots, d, \\
& (\eta-\boldsymbol{\tau}) \varphi_{i}^{\mathrm{u}}\left(\boldsymbol{p}_{i}, \boldsymbol{\tau}\right) \geq r_{\mathrm{u}}, \quad i=1, \ldots, u,
\end{aligned}
$$

which a nonconvex problem as the objective function is nonconcave while the downlink and uplink QoS constraints (47d) and (47e) are nonconvex. Like the computational approach presented in the previous sections, we now develop a lowerbounding concave approximation for the numerator of its objective function and inner convex approximations for its nonconvex constraints.

Let $\left(v^{(\kappa)}, p^{(\kappa)}, \tau^{(\kappa)}\right)$ be a feasible point for (47) found from $(\kappa-1)$ th iteration. Applying the inequalities (73) and (74) for

$$
\begin{aligned}
\varphi_{j}^{\mathrm{d}}(\boldsymbol{v}, \boldsymbol{\tau}) \geq \varphi_{j}^{\mathrm{d},(\kappa)}(\boldsymbol{v}, \boldsymbol{\tau}) \triangleq \\
\hat{a}_{j}^{\mathrm{d},(\kappa)}-\hat{b}_{j}^{\mathrm{d},(\kappa)}\left(\frac{\left|\widetilde{\lambda}_{j}\left(v_{j}^{(\kappa)}\right)\right|^{2}}{2 \Re\left\{\left(\widetilde{\lambda}_{j}\left(v_{j}^{(\kappa)}\right)\right) * \widetilde{\lambda}_{j}\left(\boldsymbol{v}_{j}\right)\right\}-\left|\widetilde{\lambda}_{j}\left(v_{j}^{(\kappa)}\right)\right|^{2}}\right. \\
\left.+\frac{v_{j}(\boldsymbol{v}, \boldsymbol{\tau})}{v_{j}\left(v^{(\kappa)}, \tau^{(\kappa)}\right)}\right),
\end{aligned}
$$

and

$$
\begin{array}{r}
\boldsymbol{\tau} \varphi_{j}^{\mathrm{d}}(\boldsymbol{v}, \boldsymbol{\tau}) \geq \hat{f}_{j}^{\mathrm{d},(\kappa)}(\boldsymbol{v}, \boldsymbol{\tau}) \triangleq \\
\hat{\hat{a}}_{j}^{\mathrm{d},(\kappa)}-\hat{\hat{b}}_{j}^{\mathrm{d},(\kappa)}\left(\frac{\left|\widetilde{\lambda}_{j}\left(v_{j}^{(\kappa)}\right)\right|^{2}}{2 \Re\left\{\left(\widetilde{\lambda}_{j}\left(v_{j}^{(\kappa)}\right)\right) * \widetilde{\lambda}_{j}\left(\boldsymbol{v}_{j}\right)\right\}-\left|\widetilde{\lambda}_{j}\left(v_{j}^{(\kappa)}\right)\right|^{2}}\right. \\
\left.+\frac{v_{j}(\boldsymbol{v}, \boldsymbol{\tau})}{v_{j}\left(v^{(\kappa)}, \tau^{(\kappa)}\right)}\right)-\frac{\hat{\hat{c}}_{j}^{\mathrm{d},(\kappa)}}{\boldsymbol{\tau}}
\end{array}
$$

over the trust region (32), where $0<\hat{a}_{j}^{\mathrm{d},(\kappa)}=$ $\varphi_{j}^{\mathrm{d}}\left(v^{(\kappa)}, \tau^{(\kappa)}\right)+2 \hat{b}_{j}^{\mathrm{d},(\kappa)}, 0<\hat{b}_{j}^{\mathrm{d},(\kappa)}=\frac{\left|\widetilde{\lambda}_{j}\left(v_{j}^{(\kappa)}\right)\right|^{2}}{v_{j}\left(v^{(\kappa)}, \tau^{(\kappa)}\right)+\left|\widetilde{\lambda}_{j}\left(v_{j}^{(\kappa)}\right)\right|^{2}}$, and $0<\hat{\hat{a}}_{j}^{\mathrm{d},(\kappa)}=2 \tau^{(\kappa)} \varphi_{j}^{\mathrm{d}}\left(v^{(\kappa)}, \tau^{(\kappa)}\right)+2 \hat{b}_{j}^{\mathrm{d},(\kappa)}, 0<$ $\hat{\hat{b}}_{j}^{\mathrm{d},(\kappa)}=\frac{\tau^{(\kappa)}\left|\widetilde{\lambda}_{j}\left(v_{j}^{(\kappa)}\right)\right|^{2}}{v_{j}\left(v^{(\kappa)}, \tau^{(\kappa)}\right)+\left|\widetilde{\lambda}_{j}\left(v_{j}^{(\kappa)}\right)\right|^{2}}, \quad 0<\hat{\hat{c}}_{j}^{\mathrm{d},(\kappa)}=$ $\left(\tau^{(\kappa)}\right)^{2} \varphi_{j}^{\mathrm{d}}\left(v^{(\kappa)}, \tau^{(\kappa)}\right)$.

Applying the inequalities (68) and (71) in the appendix for $\boldsymbol{x}=\boldsymbol{p}_{i}\left|\tilde{R}_{\mathrm{bs}}(i, i)\right|^{2}, \boldsymbol{y}=(\eta-\boldsymbol{\tau}) \sigma_{\mathrm{bs}}^{2}$ and $\bar{x}=p_{i}^{(\kappa)}\left|\tilde{R}_{\mathrm{bs}}(i, i)\right|^{2}$, $\bar{y}=\left(\eta-\tau^{(\kappa)}\right) \sigma_{\mathrm{bs}}^{2}$ yields

$$
\begin{aligned}
\varphi_{i}^{\mathrm{u}}(\boldsymbol{p}, \boldsymbol{\tau}) & \geq \varphi_{i}^{\mathrm{u},(\kappa)}(\boldsymbol{p}, \boldsymbol{\tau}) \\
& \triangleq \hat{a}_{i}^{\mathrm{u},(\kappa)}-\hat{b}_{i}^{\mathrm{u},(\kappa)}\left(\frac{p_{i}^{(\kappa)}}{\boldsymbol{p}_{i}}+\frac{\eta-\boldsymbol{\tau}}{\eta-\tau^{(\kappa)}}\right),
\end{aligned}
$$

and

$$
\begin{aligned}
(\eta-\boldsymbol{\tau}) \varphi_{i}^{\mathrm{u}}(\boldsymbol{p}, \boldsymbol{\tau}) \geq & \hat{f}_{i}^{\mathrm{u},(\kappa)}(\boldsymbol{p}, \boldsymbol{\tau}) \\
\triangleq & \hat{\hat{a}}_{i}^{\mathrm{u},(\kappa)}-\hat{\hat{b}}_{i}^{\mathrm{u},(\kappa)}\left(\frac{p_{i}^{(\kappa)}}{\boldsymbol{p}_{i}}+\frac{\eta-\boldsymbol{\tau}}{\eta-\tau^{(\kappa)}}\right) \\
& -\frac{\hat{\hat{c}}_{i}^{\mathrm{u},(\kappa)}}{\eta-\boldsymbol{\tau}},
\end{aligned}
$$

where $0<\hat{a}_{i}^{\mathrm{u},(\kappa)}=\varphi_{i}^{\mathrm{u}}\left(p^{(\kappa)}, \tau^{(\kappa)}\right)+2 \hat{b}_{i}^{\mathrm{u},(\kappa)}, 0<$ $\hat{b}_{i}^{\mathrm{u},(\kappa)}=\frac{p_{i}^{(\kappa)}\left|\tilde{R}_{\mathrm{bs}}(i, i)\right|^{2}}{\left(\eta-\tau^{(\kappa)}\right) \sigma_{\mathrm{bs}}^{2}+p_{i}^{(\kappa)}\left|\tilde{R}_{\mathrm{bs}}(i, i)\right|^{2}}$, and $0<\hat{\hat{a}}_{i}^{\mathrm{u},(\kappa)}=$ $2\left(\eta-\tau^{(\kappa)}\right) \varphi_{i}^{\mathrm{u}}\left(p^{(\kappa)}, \tau^{(\kappa)}\right)+22 \hat{\hat{b}}_{i}^{\mathrm{u},(\kappa)}, 0<\hat{\hat{b}}_{i}^{\mathrm{u},(\kappa)}=$ $\frac{\left(\eta-\tau^{(\kappa)}\right) p_{i}^{(\kappa)}\left|\tilde{R}_{\mathrm{bs}}(i, i)\right|^{2}}{\left(\eta-\tau^{(\kappa)}\right) \sigma_{\mathrm{bs}}^{2}+p_{i}^{(\kappa)}\left|\tilde{R}_{\mathrm{bs}}(i, i)\right|^{2}}, 0<\hat{\hat{c}}_{i}^{\mathrm{u},(\kappa)}=(\eta-$ $\left.\tau^{(\kappa)}\right)^{2} \varphi_{i}^{\mathrm{u}}\left(p^{(\kappa)}, \tau^{(\kappa)}\right)$

We thus solve the following convex problem to generate the next feasible point $\left(v^{(\kappa+1)}, p^{(\kappa+1)}, \tau^{(\kappa+1)}\right)$ for (47):

$$
\begin{array}{r}
\max _{0<\boldsymbol{\tau}<1, \boldsymbol{v}, \boldsymbol{p}} \hat{L}^{(\kappa)}(\boldsymbol{v}, \boldsymbol{p}, \boldsymbol{\tau}) \triangleq \sum_{j=1}^{d} \hat{f}_{j}^{\mathrm{d},(\kappa)}(\boldsymbol{v}, \boldsymbol{\tau}) \\
+\sum_{i=1}^{u} \hat{f}_{i}^{\mathrm{u},(\kappa)}(\boldsymbol{p}, \boldsymbol{\tau})-\hat{\Phi}\left(v^{(\kappa)}, p^{(\kappa)}, \tau^{(\kappa)}\right) P^{t o t}(\boldsymbol{v}, \boldsymbol{p}) \\
\text { s.t. } \quad(32),(47 b),(47 c), \\
\varphi_{j}^{\mathrm{d},(\kappa)}(\boldsymbol{v}, \boldsymbol{\tau}) \geq r_{\mathrm{d}} / \boldsymbol{\tau}, \quad j=1, \ldots, d, \\
\varphi_{i}^{\mathrm{u},(\kappa)}\left(\boldsymbol{p}_{i}, \boldsymbol{\tau}\right) \geq r_{\mathrm{u}} /(\eta-\boldsymbol{\tau}), \quad i=1, \ldots, u,
\end{array}
$$

where by (49) and (51) the first two terms in (52a) provide a lower-bounding concave approximation for the numerator of the objective function in (47a), while by (48) and (50) the convex constraints $(52 \mathrm{~b})$ and (52c) provides an inner convex approximation for the nonconvex constraint (47d) and (47e), respectively. Similarly to (38), it is easy to show that

$$
\hat{\Phi}\left(v^{(\kappa+1)}, p^{(\kappa+1)}, \tau^{(\kappa+1)}\right)>\hat{\Phi}\left(v^{(\kappa)}, p^{(\kappa)}, \tau^{(\kappa)}\right),
$$

as far as $\left(v^{(\kappa+1)}, p^{(\kappa+1)}, \tau^{(\kappa+1)}\right) \neq\left(v^{(\kappa)}, p^{(\kappa)}, \tau^{(\kappa)}\right)$, i.e. $\left(v^{(\kappa+1)}, p^{(\kappa+1)}, \tau^{(\kappa+1)}\right)$ is a better feasible point than $\left(v^{(\kappa)}, p^{(\kappa)}, \tau^{(\kappa)}\right)$ for the nonconvex optimization problem (47). The sequence $\left\{\left(v^{(\kappa)}, p^{(\kappa)}, \tau^{(\kappa)}\right)\right\}$ thus converges at least to a locally optimal solution of (47).

To locate an initial feasible point $\left(v^{(0)}, p^{(0)}, \tau^{(0)}\right)$ for (47) we fix $\tau^{(0)}$ and replace $\left|\tilde{\lambda}_{j}\left(\boldsymbol{v}_{j}\right)\right|^{2}$ by $\left(\bar{\lambda}_{j}\left(\boldsymbol{v}_{j}\right)\right)^{2}$ for $\bar{\lambda}_{j}\left(\boldsymbol{v}_{j}\right)=$ $\Re\left\{\tilde{\lambda}_{j}\left(\boldsymbol{v}_{j}\right)\right\}$ in (43), to make

$$
\varphi_{j}^{\mathrm{d}}(\boldsymbol{v}, \boldsymbol{\tau})=\ln \left(1+\frac{\left(\bar{\lambda}_{j}\left(\boldsymbol{v}_{j}\right)\right)^{2}}{v_{j}(\boldsymbol{v}, \boldsymbol{\tau})}\right),
$$

so the nonconvex downlink QoS constraint (47d) is equivalent to the SOC constraint

$$
\begin{aligned}
\bar{\lambda}_{j}\left(\boldsymbol{v}_{j}\right) & \geq \sqrt{e^{r_{j}^{\min } / \tau^{(0)}}-1} \sqrt{v_{j}(\boldsymbol{v}, \boldsymbol{\tau})} \\
& =\sqrt{e^{r_{j}^{\min } / \tau^{(0)}}-1} \| \begin{array}{c}
\left(h_{j, \text { bs }}^{H} \boldsymbol{v}_{\ell}\right)_{\ell \in \mathcal{D} \backslash\{j\}} \|_{0} \sigma_{\mathrm{d}} \\
\tau_{2}
\end{array}
\end{aligned}
$$

while the nonconvex uplink QoS constraint (47e) is also equivalent to the following SOC constraint

$$
\begin{array}{r}
\sqrt{\boldsymbol{p}_{i}}\left|\tilde{R}_{\mathrm{bs}}(i, i)\right| \geq \sqrt{e^{r_{\mathrm{bs}}^{\min } /\left(\eta-\tau^{(0)}\right)}-1} \sqrt{\left(\eta-\tau^{(0)}\right)} \sigma_{\mathrm{bs}}, \\
i=1, \ldots, u .
\end{array}
$$


We then solve the convex problem

$$
\min _{\boldsymbol{v}, \boldsymbol{p}} \sum_{j=1}^{d}\left\|\boldsymbol{v}_{j}\right\|^{2}+\sum_{i=1}^{u} \boldsymbol{p}_{i} \quad \text { s.t. } \quad(47 b),(47 c),(53),(54)
$$

to obtain a feasible point $\left(v^{(0)}, p^{(0)}, \tau^{(0)}\right)$ for (47).

Algorithm 3 summarizes our proposed computational procedure for solving (47).

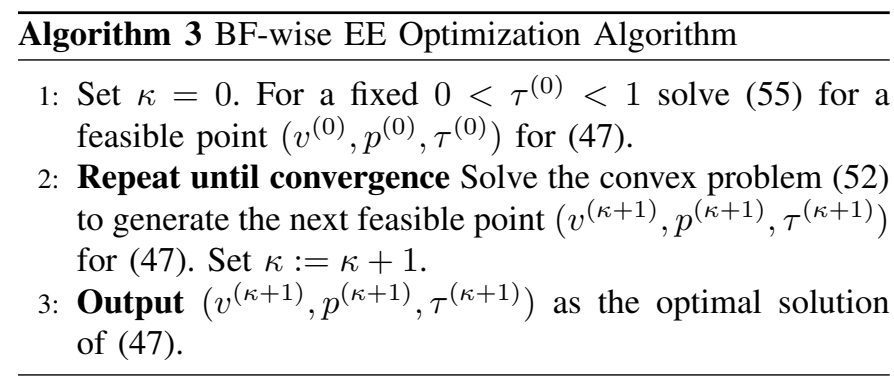

\section{NumERICAL RESUltS}

The data of wireless communications in a macrocell environment is used to weight the pros and con of the proposed STRs. Table I taken from [35] lists important parameters used in all simulations. The number of served downlink users and uplink users are $d=u=n / 2$, which also means $n_{1}=n_{2}=n / 2$. For e-FT and FB, set $\epsilon=0.1$. 1 The channel vector between the BS and an user follows the line for sight (LOS) path loss model as $10^{-P L_{\mathrm{LOS}} / 20} \tilde{h}$ with $P L_{\mathrm{LOS}}=103.4+24.2 \log _{10} \delta_{1}$, where the entries of $\tilde{h}$ are independent circularly-symmetric Gaussian random variables with zero means and unit variance and $\delta_{1}(\mathrm{~km})$ is their distance. The interfering channel from an UL to a DL follows the non-line-of-sight (NLOS) path loss model as $10^{-P L_{\mathrm{NLOS}} / 20} \tilde{h}_{u u}$ with $P L_{\mathrm{NLOS}}=131.1+42.8 \log _{10} \delta_{2}$, where $\tilde{h}_{u u}$ is circular-symmetric random variable with zero means and unit variance, and $\delta_{2}$ is their distance. If not specially specified, DL and UL users are uniformly distributed in the cell so that $\delta_{1}=0.25 \mathrm{~km}$ and $\delta_{2}=0.1 \mathrm{~km}$, respectively.

TABLE I: Simulation Parameters

\begin{tabular}{|c|c|}
\hline Parameter & Value \\
\hline Carrier frequency & $2 \mathrm{GHz}$ \\
System bandwidth & $10 \mathrm{MHz}$ \\
Maximum BS transmit power $\left(P_{\mathrm{BS}}^{\max }\right)$ & $46 \mathrm{dBm}$ \\
Maximum user transmit power $\left(P_{\mathrm{UE}}^{\max }\right)$ & $23 \mathrm{dBm}$ \\
Dynamic circuit power of the BS $\left(P_{c}^{B S}\right)$ & $6.31 \mathrm{~W}$ \\
Static circuit power of the BS $\left(P_{s}^{B S}\right)$ & $0.5012 \mathrm{~W}$ \\
Dynamic circuit power of a UE $\left(P_{c}^{U E}\right)$ & $4.417 \mathrm{~W}$ \\
Static circuit power of the UEs $\left(P_{s}^{U E}\right)$ & $0.1 \mathrm{~W}$ \\
Noise power density & $-174 \mathrm{dBm} / \mathrm{Hz}$ \\
\hline
\end{tabular}

The tolerance for the algorithm convergence is set to $10^{-4}$. In arriving at the final figures, the results of 1000 Monte-Carlo runs are averaged.

\footnotetext{
${ }^{1}$ According [36], the one time slot is $0.667 \mathrm{~ms}$ and the antennas switching time is $0.02 \mathrm{~ms}$ while the guard band is also about $10 \%$ of the available bandwidth.
}

\section{A. Spectral efficiency in terms of max-min throughput}

Although the paper is mainly focused on EE optimization subject to downlink and uplink QoS constraints, it is still of interest to know how the spectral efficiency of the three STRs by considering the following max-min throughput optimization problem

$$
\begin{array}{r}
\max _{\boldsymbol{v}, \boldsymbol{p}} \min \left\{\min _{j=1, \ldots, d} r_{j}^{\mathrm{d}}(\boldsymbol{v}, \boldsymbol{p}), \min _{i=1, \ldots, u} r_{i}^{\mathrm{u}}\left(\boldsymbol{v}, \boldsymbol{p}_{i}\right)\right\} \\
\text { s.t. } \quad(10 b),(10 c)
\end{array}
$$

for the FD-based STR, and

$$
\begin{array}{r}
\max _{0<\boldsymbol{\tau}<1, \widetilde{\boldsymbol{v}}, \boldsymbol{p}} \min \left\{\min _{j=1, \ldots, d} \boldsymbol{\tau} \rho_{j}^{\mathrm{d}}(\widetilde{\boldsymbol{v}}), \min _{i=1, \ldots, u}(\eta-\boldsymbol{\tau}) \rho_{i}^{\mathrm{u}}\left(\boldsymbol{p}_{i}\right)\right\} \\
\text { s.t. } \quad(26 b),(26 c),(26 f),(26 g)
\end{array}
$$

for the TF-wise STR, and

$$
\begin{array}{r}
\max _{0<\boldsymbol{\tau}<1, \boldsymbol{v}, \boldsymbol{p}} \min \left\{\min _{j=1, \ldots, d} \boldsymbol{\tau} \sum_{j=1}^{d} \varphi_{j}^{\mathrm{d}}(\boldsymbol{v}, \boldsymbol{\tau}),\right. \\
\left.\min _{i=1, \ldots, u}(\eta-\boldsymbol{\tau}) \varphi_{i}^{\mathrm{u}}\left(\boldsymbol{p}_{i}, \boldsymbol{\tau}\right)\right\} \quad \text { s.t. } \quad(47 b),(47 c),
\end{array}
$$

for the BF-wise STR. Obviously, these nonsmooth nonconvex optimization problems can be solved by the Algorithms that are similar to Algorithms 1-3, which at the $\kappa$ th iteration compute the following convex problem

$$
\begin{array}{r}
\max _{\boldsymbol{v}, \boldsymbol{p}} \min \left\{\min _{j=1, \ldots, d} r_{j}^{\mathrm{d},(\kappa)}(\boldsymbol{v}, \boldsymbol{p}), \min _{i=1, \ldots, u} r_{i}^{\mathrm{u},(\kappa)}\left(\boldsymbol{v}, \boldsymbol{p}_{i}\right)\right\} \\
\text { s.t. } \quad(10 b),(10 c),(16),(18),
\end{array}
$$

with $r_{j}^{\mathrm{d},(\kappa)}$ and $r_{i}^{\mathrm{u},(\kappa)}$ defined from (15) and (17) to generate a better feasible point $\left(v^{(\kappa+1)}, p^{(\kappa+1)}\right)$ for (56), and

$$
\begin{array}{r}
\max _{\widetilde{\boldsymbol{v}}, \boldsymbol{p}, \boldsymbol{\theta}, \boldsymbol{\tau}} \min \left\{\min _{j=1, \ldots, d} \tilde{f}_{j}^{\mathrm{d},(\kappa)}\left(\widetilde{\boldsymbol{v}}, \boldsymbol{\theta}_{1}\right), \min _{i=1, \ldots, u} \tilde{f}_{i}^{\mathrm{u},(\kappa)}\left(\boldsymbol{x}_{i}, \boldsymbol{\theta}_{2}\right)\right\} \\
\text { s.t. } \quad(26 f),(26 g),(27),(28 b),(32),
\end{array}
$$

with $\tilde{f}_{j}^{\mathrm{d},(\kappa)}$ and $\tilde{f}_{i}^{\mathrm{u},(\kappa)}$ defined from (31) and (35) to generate a better feasible point $\left(v^{(\kappa+1)}, p^{(\kappa+1)}, \theta^{(\kappa+1)}, \tau^{(\kappa+1)}\right)$ for $(57)$, and

$$
\begin{array}{r}
\max _{0<\boldsymbol{\tau}<1, \boldsymbol{v}, \boldsymbol{p}} \min \left\{\min _{j=1, \ldots, d} \hat{f}_{j}^{\mathrm{d},(\kappa)}(\boldsymbol{v}, \boldsymbol{\tau}),\right. \\
\left.\min _{i=1, \ldots, u} \hat{f}_{i}^{\mathrm{u},(\kappa)}(\boldsymbol{p}, \boldsymbol{\tau})\right\} \\
\quad \text { s.t. } \quad(32),(47 b),(47 c)
\end{array}
$$

with $\hat{f}_{j}^{\mathrm{d},(\kappa)}$ and $\hat{f}_{i}^{\mathrm{u},(\kappa)}$ defined from (49) and (51) to generate a better feasible point $\left(v^{(\kappa+1)}, p^{(\kappa+1)}, \tau^{(\kappa+1)}\right)$ for (58).

Fig. 4 plots the achievable minimum throughput under different $n$. Only the achievable max-min throughput of the FD based STR is severely downgraded when the SI $\sigma_{S I}^{2}$ increases, which is especially low for the practical range $[-120,-90] \mathrm{dB}$ of $\sigma_{S I}^{2}$. Both TF and BF outperform FD for $\sigma_{S I}^{2} \geq-110 \mathrm{~dB}$. At $\sigma_{S I}^{2}=-90 \mathrm{~dB}$, the achievable minimum throughput by the formers is actually twice of that achievable by the latter. The gap is wider by increasing the number of transmit antennas, which leads to more downlink interference to uplinks under FD.

Fig. 5 shows the effectiveness of the optimal time fraction as e-TF clearly outperforms the half-duplex (HD) TDD, which use all $n$ antennas and $(1-\eta) / 2$ fraction of the time slot for 


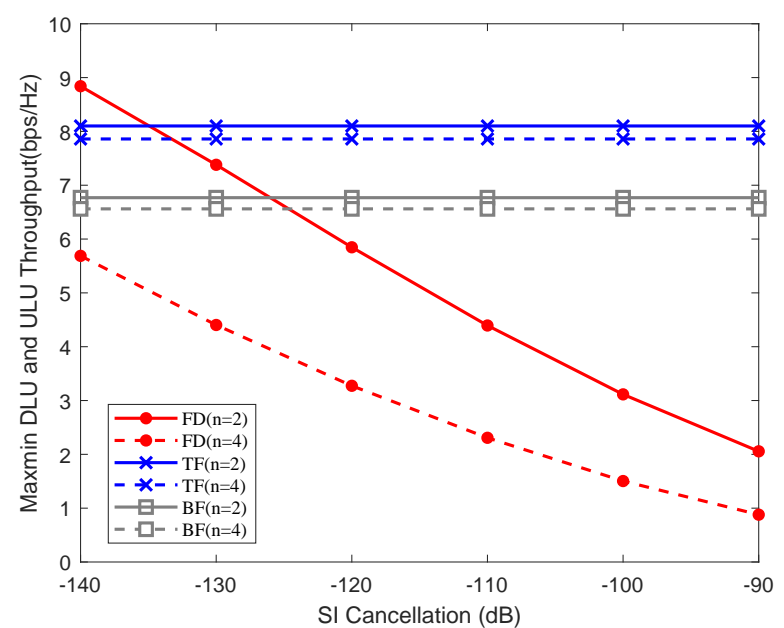

(a)

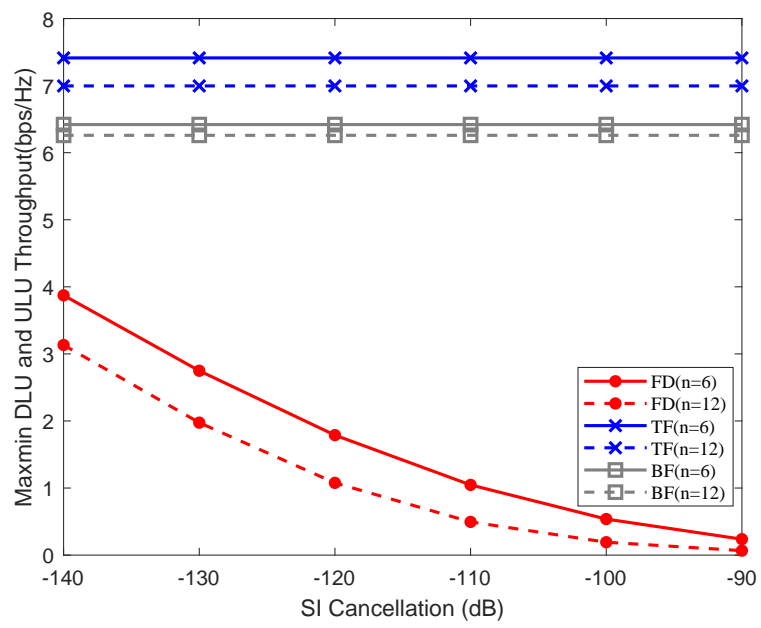

(b)

Fig. 4: Achievable minimum DLU and ULU throughput vs SI $\sigma_{S I}^{2}$

each downlink and uplink service. It also shows the benefit of employing all antennas for both downlink and uplink services, as TF is not only consistently outperformed by e-TF but it is also outperformed by HD TDD with $n \geq 6$. It is not surprised that the HD FDD, which allocates $(1-\eta) / 2$ bandwidth' portion for each downlink and uplink service, is the worst performer.

Table II provides the rounded average number of iterations for the results in Fig. 4, which particularly shows that all the three algorithms converge within 25 iterations.

Furthermore, to examine the impact of UL users to DL users in the FD-based STR we also simulate the following scenario: DL users are still uniformly distributed over the macrocell but there is one UL user in proximity of each DL user. Table III shows the achievable minimum throughput by the FD-based STRs for $n=6$, which is very sensitive to the distance $\delta_{2}$ between a DL user and its nearest UL user. The ratio of that between that achieved for $\delta_{2}=0.05 \mathrm{~km}$ and that achieved for

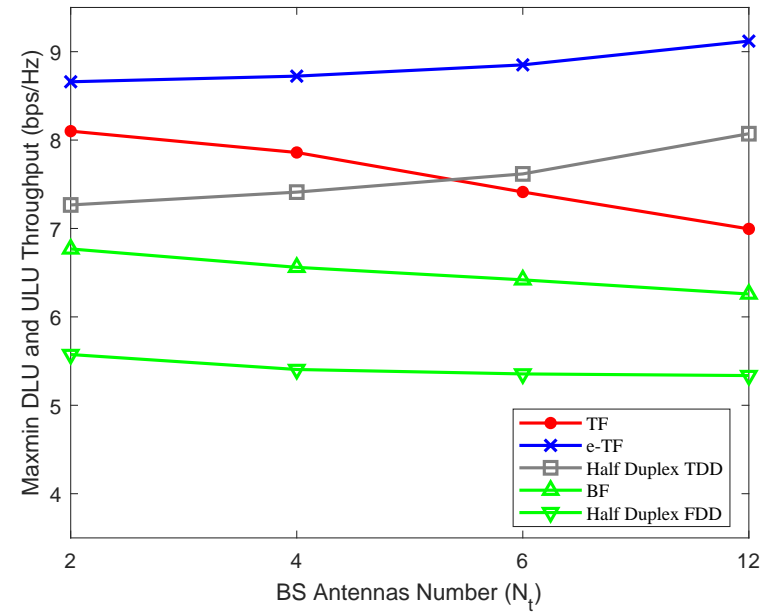

Fig. 5: Achievable minimum DLU and ULU throughput vs BS antennas number $N_{t}$

TABLE II: The rounded average number of iterations for implementing the max-min-throughput Algorithms

\begin{tabular}{|c|c|c|c|c|}
\hline & $n=2$ & $n=4$ & $n=6$ & $n=12$ \\
\hline FD-based STR $\left(\delta_{S I}^{2}=-140 \mathrm{~dB}\right)$ & 6 & 8 & 10 & 14 \\
\hline FD-based STR $\left(\delta_{S I}^{2}=-130 \mathrm{~dB}\right)$ & 7 & 9 & 11 & 15 \\
\hline FD-based STR $\left(\delta_{S I}^{2}=-120 \mathrm{~dB}\right)$ & 7 & 10 & 15 & 16 \\
\hline FD-based STR $\left(\delta_{S I}^{2}=-110 \mathrm{~dB}\right)$ & 8 & 11 & 17 & 17 \\
\hline FD-based STR $\left(\delta_{S I}^{2}=-100 \mathrm{~dB}\right)$ & 10 & 12 & 18 & 20 \\
\hline FD-based STR $\left(\delta_{S I}^{2}=-90 \mathrm{~dB}\right)$ & 11 & 12 & 21 & 22 \\
\hline $\operatorname{TF}-w i s e$ STR & 5 & 7 & 12 & 12 \\
\hline e-TF-wise STR & 6 & 7 & 11 & 12 \\
\hline BF-wise STR & 4 & 10 & 12 & 16 \\
\hline
\end{tabular}

$\delta_{2}=0.005 \mathrm{~km}$ is more than ten and increases as the SI $\sigma_{S I}^{2}$ increases.

TABLE III: Achievable minimum throughput of FD-based STR (bps/Hz) under different $\delta_{2}$ and $\sigma_{S I}^{2}$

\begin{tabular}{|c|c|c|c|c|}
\hline$\delta_{S I}^{2} \delta_{2}$ & $0.05 \mathrm{~km}$ & $0.03 \mathrm{~km}$ & $0.01 \mathrm{~km}$ & $0.005 \mathrm{~km}$ \\
\hline$-140 \mathrm{~dB}$ & 3.1125 & 2.3542 & 0.9213 & 0.3214 \\
\hline$-130 \mathrm{~dB}$ & 2.0376 & 1.6678 & 0.5033 & 0.2392 \\
\hline$-120 \mathrm{~dB}$ & 1.4424 & 0.9874 & 0.1779 & 0.0884 \\
\hline$-110 \mathrm{~dB}$ & 0.8431 & 0.4879 & 0.0621 & 0.0297 \\
\hline$-100 \mathrm{~dB}$ & 0.4101 & 0.1892 & 0.0203 & 0.0096 \\
\hline$-90 \mathrm{~dB}$ & 0.1740 & 0.0676 & 0.0065 & 0.0031 \\
\hline
\end{tabular}

\section{B. EE optimization}

The downlink and uplink throughput thresholds for the QoS are set as $r_{\mathrm{d}}=0.4 \mathrm{bps} / \mathrm{Hz}$ and $r_{\mathrm{u}}=1 \mathrm{bps} / \mathrm{Hz}$, while the circuit power at the BS and ULs are $P_{\mathrm{BS}}=n P_{c}^{B S}+P_{s}^{B S} \mathrm{~W}$ and $P_{\mathrm{UE}}=u P_{c}^{U E}+P_{s}^{U E} \mathrm{~W}$, and the drain efficiency of power amplifier is $\zeta=20 \%$ [37].

Fig. 6 shows the achievable EE by Algorithms 1-3 under various numbers $(n)$ of $\mathrm{BS}$ antennas. As expected, the achievable EE by the FD-based STR is quickly dropped when the SI level $\sigma_{S I}^{2}$ raises up. At $\sigma_{S I}^{2}=-90 \mathrm{~dB}$, the achievable $\mathrm{EE}$ by the latter becomes double of that by the former. TF and e-TF are seen more efficient than BF. Furthermore, e-TF 
outperforms TF thanks to its its exploitation of all available antennas for its STR. As it happens to the spectral efficiency, using more antennas in serving more users makes the FD's EE deteriorated but improves the EE of other STRs. They perform similarly at low SI level $\sigma_{S I}^{2} \in[-140,-120] \mathrm{dB}$ but the FD is quickly outperformed by others for $\sigma_{S I}^{2} \geq-110 \mathrm{~dB}$.

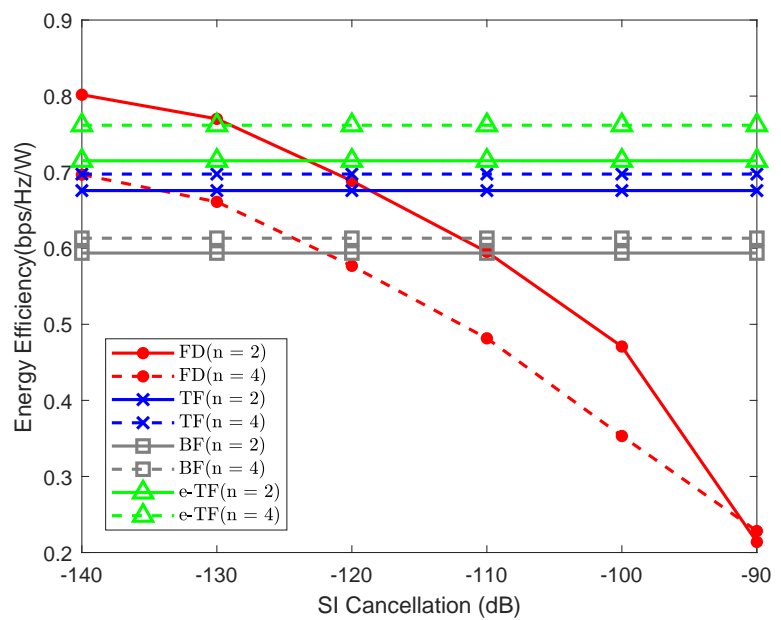

(a)

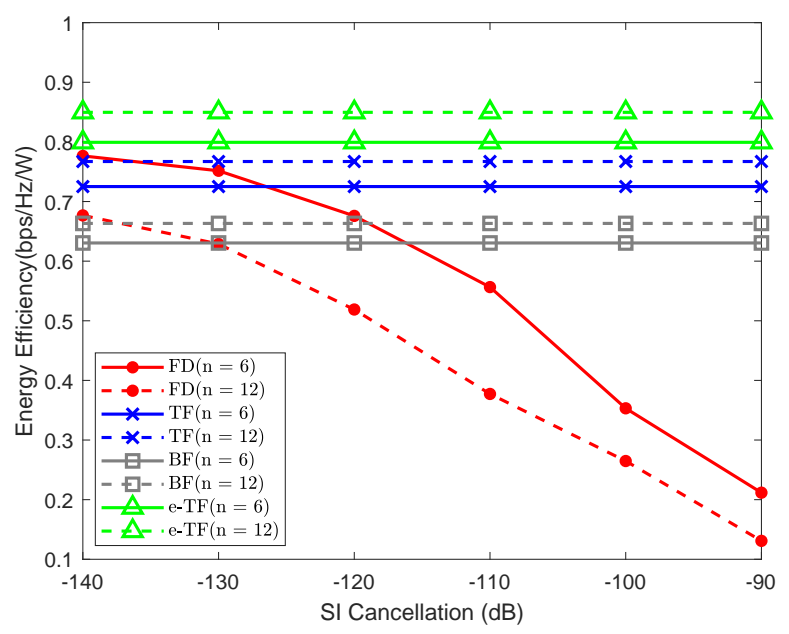

(b)

Fig. 6: Achievable EE by three STRs

The rounded average number of algorithm iterations to output Fig. 6 is given by Table IV. All the proposed algorithms are seen convergent within 45 iterations. Compared to Table II, a few more iterations are needed, which are quite expected because the EE optimization problem is seen more complex than the spectral efficiency optimization problem. Both Algorithms 2 and 3 exploit well the fractional structure of the objective function without its direct approximation. All the percentage number in the tables represent the probability of obtaining a valid result in all Monte-Carlo runs. The success percentage of the FD-based STR is extremely low for $\sigma_{S I}^{2} \in[-100,-90]$ $\mathrm{dB}$ as it hardly provides the required QoS. In contrast, the other STRs always perform well with hundred percentage of success as they can avoid the FD inherent self-interference.

\section{EE in serving doubled number of users}

Until now, the e-TF and HD-TDD are purposely allowed to serve $n / 2$ downlink users and $n / 2$ uplink users to accommodate comparison with other STRs. Table V provides their achievable spectral efficiency and EE in serving double numbers of both downlink and uplink users ( $n$ downlink users and $n$ uplink users). The rounded averaged number of iterations to output the EE of e-TF is given by the last row of Table IV. It is reasonable to expect that their achievable EE is higher compared to that achievable by serving $n / 2$ donwlink users and $n / 2$ uplink users under the same power constraints.

\section{Conclusions}

The paper has proposed three possible techniques for implementing heterogeneous macrocell downlink and uplink communications within one time-slot, which are the FD-based STR, fraction-time-wise STR, and bandwidth-fraction-wise STR. The three optimization algorithms have been developed to compute the energy efficiency by joint downlink beamforming and uplink power allocation under these STRs. As expected, the FD hardly suitable as its inherent self-interference is not expected to be suppressed to the level for proper implementation. Being free from the mutual interference between downlinks and uplinks, other two techniques have been shown to be effective for STR. Especially, the fraction-time-wise STR is very efficient and can serve many more downlinks and uplinks as it can exploit the full number of the BS antennas for STR. Both the fraction-time base STR and fraction-bandwidth STR for multi-cell result in separated down-link multi-cell systems and uplink multi-cell systems, so they can be used in realistic cellular networks. Using improper Gaussian signaling instead of the conventional proper Gaussian signaling in STR is under current study.

\section{APPENDIX: RATE FUNCTION APPROXIMATION}

The following result [19] is used.

Theorem 1: The following inequality holds true

$$
\begin{aligned}
& \frac{\ln (1+1 / \boldsymbol{x} \boldsymbol{y})}{\boldsymbol{t}} \geq 2 \frac{\ln (1+1 / \bar{x} \bar{y})}{\bar{t}} \\
&+\frac{1}{(\bar{x} \bar{y}+1) \bar{t}}\left(2-\frac{\boldsymbol{x}}{\bar{x}}-\frac{\boldsymbol{y}}{\bar{y}}\right)-\frac{\ln (1+1 / \bar{x} \bar{y})}{\bar{t}^{2}} \boldsymbol{t} \\
& \quad \forall(\boldsymbol{x}, \boldsymbol{y}, \boldsymbol{t}) \in \mathbb{R}_{+}^{3},(\bar{x}, \bar{y}, \bar{t}) \in \mathbb{R}_{+}^{3} .
\end{aligned}
$$

The right-hand-side (RHS) of (62) agrees with the left-handside (LHS) at $(\bar{x}, \bar{y}, \bar{t})$.

A particular case of (62) is

$$
\begin{array}{r}
\ln (1+1 / \boldsymbol{x} \boldsymbol{y}) \geq \ln (1+1 / \bar{x} \bar{y})+\frac{1}{(\bar{x} \bar{y}+1)}\left(2-\frac{\boldsymbol{x}}{\bar{x}}-\frac{\boldsymbol{y}}{\bar{y}}\right) \\
\forall(\boldsymbol{x}, \boldsymbol{y}) \in \mathbb{R}_{+}^{2},(\bar{x}, \bar{y}) \in \mathbb{R}_{+}^{2},
\end{array}
$$

and

$$
\begin{array}{r}
\frac{\ln (1+1 / \boldsymbol{x})}{\boldsymbol{t}} \geq 2 \frac{\ln (1+1 / \bar{x})}{\bar{t}}+\frac{1}{(\bar{x}+1) \bar{t}}\left(1-\frac{\boldsymbol{x}}{\bar{x}}\right) \\
-\frac{\ln (1+1 / \bar{x})}{\bar{t}^{2}} \boldsymbol{t} \forall(\boldsymbol{x}, \boldsymbol{t}) \in \mathbb{R}_{+}^{2}, \quad(\bar{x}, \bar{t}) \in \mathbb{R}_{+}^{2},
\end{array}
$$


TABLE IV: The rounded average number of iterations for the convergence of Algorithms 1-3

\begin{tabular}{|c|c|c|c|c|}
\hline & $n=2$ & $n=4$ & $n=6$ & $n=12$ \\
\hline FD-based STR $\left(\sigma_{S I}^{2}=-140 \mathrm{~dB}\right)$ & $16(100 \%)$ & $19(100 \%)$ & $16(100 \%)$ & $27(100 \%)$ \\
\hline FD-based STR $\left(\sigma_{S I}^{2}=-130 \mathrm{~dB}\right)$ & $17(100 \%)$ & $20(100 \%)$ & $22(100 \%)$ & $22(100 \%)$ \\
\hline FD-based STR $\left(\sigma_{S I}^{2}=-120 \mathrm{~dB}\right)$ & $19(99 \%)$ & $23(100 \%)$ & $27(94 \%)$ & $34(100 \%)$ \\
\hline FD-based STR $\left(\sigma_{S I}^{2}=-110 \mathrm{~dB}\right)$ & $20(99 \%)$ & $28(96 \%)$ & $34(88 \%)$ & $40(75 \%)$ \\
\hline FD-based STR $\left(\sigma_{S I}^{2}=-100 \mathrm{~dB}\right)$ & $22(66 \%)$ & $35(75 \%)$ & $35(69 \%)$ & $38(50 \%)$ \\
\hline FD-based STR $\left(\sigma_{S I}^{2}=-90 \mathrm{~dB}\right)$ & $25(2 \%)$ & $35(17 \%)$ & $38(3 \%)$ & $44(6 \%)$ \\
\hline TF-wise STR & $14(100 \%)$ & $11(100 \%)$ & $10(100 \%)$ & $10(100 \%)$ \\
\hline e-TF-wise STR & $12(100 \%)$ & $9(100 \%)$ & $10(100 \%)$ & $9(100 \%)$ \\
\hline BF-wise STR & $19(100 \%)$ & $10(100 \%)$ & $22(100 \%)$ & $18(100 \%)$ \\
\hline e-TF-wise STR (double users) & $13(100 \%)$ & $11(100 \%)$ & $12(100 \%)$ & $11(100 \%)$ \\
\hline
\end{tabular}

TABLE V: Achievable spectral efficiency (SE) in terms of achieved minimum throughput $(\mathrm{bps} / \mathrm{Hz})$ and $\mathrm{EE}(\mathrm{bps} / \mathrm{Hz} / \mathrm{W})$ in serving double users vs BS antennas number $n$

\begin{tabular}{|c|c|c|c|c|}
\hline & $n=2$ & $n=4$ & $n=6$ & $n=12$ \\
\hline e-TF STR's SE & 7.6310 & 6.9935 & 6.7696 & 6.7040 \\
\hline HD TDD's SE & 6.3821 & 5.9489 & 5.8242 & 5.5425 \\
\hline e-TF STR's EE & 1.0232 & 1.0996 & 1.1352 & 1.1996 \\
\hline HD TDD's EE & 0.9393 & 0.9964 & 0.9996 & 1.0711 \\
\hline
\end{tabular}

and

$$
\begin{aligned}
\ln (1+1 / \boldsymbol{x}) \geq \ln (1+1 / \bar{x}) & +\frac{1}{(\bar{x}+1)}\left(1-\frac{\boldsymbol{x}}{\bar{x}}\right) \\
& \forall \boldsymbol{x} \in \mathbb{R}_{+}^{2}, \bar{x} \in \mathbb{R}_{+}^{2} .
\end{aligned}
$$

Another inequality

$$
\frac{\boldsymbol{x}}{\boldsymbol{t}} \geq 2 \frac{\sqrt{\bar{x}} \sqrt{x}}{\bar{t}}-\frac{\bar{x}}{\bar{t}^{2}} \boldsymbol{t} \quad \forall \boldsymbol{x}>0, \boldsymbol{t}>0, \bar{x}>0, \bar{t}>0
$$

follows from the convexity of the function $x / t=\sqrt{x}^{2} / t$ on $\sqrt{x}$ and $t$. to

Substituting $x \rightarrow 1 / x$ and $\bar{x} \rightarrow 1 / \bar{x}$ in (62) and (63) lead

$$
\begin{gathered}
\frac{\ln (1+\boldsymbol{x} / \boldsymbol{y})}{\boldsymbol{t}} \geq 2 \frac{\ln (1+\bar{x} / \bar{y})}{\bar{t}}+-\frac{\bar{x}}{(\bar{y}+\bar{x}) \bar{t}}\left(2-\frac{\bar{x}}{\boldsymbol{x}}\right. \\
\left.-\frac{\boldsymbol{y}}{\bar{y}}\right)-\frac{\ln (1+\bar{x} / \bar{y})}{\bar{t}^{2}} \boldsymbol{t} \quad \forall(\boldsymbol{x}, \boldsymbol{y}, \boldsymbol{t}) \in \mathbb{R}_{+}^{3},(\bar{x}, \bar{y}, \bar{t}) \in \mathbb{R}_{+}^{3},
\end{gathered}
$$

and

$$
\begin{array}{r}
\ln (1+\boldsymbol{x} / \boldsymbol{y}) \geq \ln (1+\bar{x} / \bar{y})+\frac{\bar{x}}{\bar{y}+\bar{x}}\left(2-\frac{\bar{x}}{\boldsymbol{x}}-\frac{\boldsymbol{y}}{\bar{y}}\right) \\
\forall(\boldsymbol{x}, \boldsymbol{y}) \in \mathbb{R}_{+}^{2},(\bar{x}, \bar{y}) \in \mathbb{R}_{+}^{2} .
\end{array}
$$

As a particular case of (67) and (68) it is

$$
\begin{aligned}
& \frac{\ln (1+\boldsymbol{x})}{\boldsymbol{t}} \geq 2 \frac{\ln (1+\bar{x})}{\bar{t}}+\frac{\bar{x}}{(\bar{x}+1) \bar{t}}\left(1-\frac{\bar{x}}{\boldsymbol{x}}\right) \\
& \quad-\frac{\ln (1+\bar{x})}{\bar{t}^{2}} \boldsymbol{t} \quad \forall(\boldsymbol{x}, \boldsymbol{t}) \in \mathbb{R}_{+}^{2},(\bar{x}, \bar{t}) \in \mathbb{R}_{+}^{2},
\end{aligned}
$$

and

$$
\begin{array}{r}
\ln (1+\boldsymbol{x}) \geq \ln (1+\bar{x})+\frac{\bar{x}}{\bar{x}+1}\left(1-\frac{\bar{x}}{\boldsymbol{x}}\right) \\
\forall x \in \mathbb{R}_{+}, \bar{x} \in \mathbb{R}_{+} .
\end{array}
$$

Substituting $t \rightarrow 1 / t$ and $\bar{t} \rightarrow 1 / \bar{t}$ in (67) leads to

$$
\begin{aligned}
& \boldsymbol{t} \ln (1+\boldsymbol{x} / \boldsymbol{y}) \geq 2 \bar{t} \ln (1+\bar{x} / \bar{y})+\frac{\bar{t} \bar{x}}{\bar{y}+\bar{x}}\left(2-\frac{\bar{x}}{\boldsymbol{x}}-\right. \\
& \left.\frac{\boldsymbol{y}}{\bar{y}}\right)-\frac{\bar{t}^{2} \ln (1+\bar{x} / \bar{y})}{\boldsymbol{t}} \quad \forall(\boldsymbol{x}, \boldsymbol{y}, \boldsymbol{t}) \in \mathbb{R}_{+}^{3},(\bar{x}, \bar{y}, \bar{t}) \in \mathbb{R}_{+}^{3},
\end{aligned}
$$

By substituting $\boldsymbol{x} \rightarrow\|\boldsymbol{x}\|^{2}$ and $\bar{x} \rightarrow\|\bar{x}\|^{2}$ with $\boldsymbol{x} \in \mathbb{C}^{n}$ and $\bar{x} \in \mathbb{C}^{n}$ in (67), (68), and (71) and using the inequality

$$
\|\boldsymbol{x}\|^{2} \geq 2 \Re\left\{\bar{x}^{H} \boldsymbol{x}\right\}-\|\bar{x}\|^{2} \quad \forall \boldsymbol{x} \in \mathbb{C}^{n}, \bar{x} \in \mathbb{C}^{n}
$$

we obtain

$$
\begin{gathered}
\frac{\ln \left(1+\|\boldsymbol{x}\|^{2} / \boldsymbol{y}\right)}{\boldsymbol{t}} \geq 2 \frac{\ln \left(1+\|\bar{x}\|^{2} / \bar{y}\right)}{\bar{t}}+\frac{\|\bar{x}\|^{2}}{\left(\bar{y}+\|\bar{x}\|^{2}\right) \bar{t}}(2 \\
\left.-\frac{\|\bar{x}\|^{2}}{2 \Re\left\{\bar{x}^{H} \boldsymbol{x}\right\}-\|\bar{x}\|^{2}}-\frac{\boldsymbol{y}}{\bar{y}}\right)-\frac{\ln \left(1+\|\bar{x}\|^{2} / \bar{y}\right)}{\bar{t}^{2}} \boldsymbol{t} \\
\quad \forall(\boldsymbol{x}, \boldsymbol{y}, \boldsymbol{t}) \in \mathbb{C}^{n} \times \mathbb{R}_{+}^{2},(\bar{x}, \bar{y}, \bar{t}) \in \mathbb{C}^{n} \times \mathbb{R}_{+}^{2},
\end{gathered}
$$

and

$$
\begin{aligned}
& \ln \left(1+\|\boldsymbol{x}\|^{2} / \boldsymbol{y}\right) \geq \ln \left(1+\|\bar{x}\|^{2} / \bar{y}\right) \\
& +\frac{\|\bar{x}\|^{2}}{\bar{y}+\|\bar{x}\|^{2}}\left(2-\frac{\|\bar{x}\|^{2}}{2 \Re\left\{\bar{x}^{H} \boldsymbol{x}\right\}-\|\bar{x}\|^{2}}-\frac{\boldsymbol{y}}{\bar{y}}\right) \\
& \quad \forall(\boldsymbol{x}, \boldsymbol{y}) \in \mathbb{C}^{n} \times \mathbb{R}_{+}, \quad(\bar{x}, \bar{y}) \in \mathbb{C}^{n} \times \mathbb{R}_{+} .
\end{aligned}
$$

and

$$
\begin{aligned}
& \boldsymbol{t} \ln \left(1+\|\boldsymbol{x}\|^{2} / \boldsymbol{y}\right) \geq 2 \bar{t} \ln \left(1+\|\bar{x}\|^{2} / \bar{y}\right) \\
& \quad+\frac{\bar{t}\|\bar{x}\|^{2}}{\bar{y}+\|\bar{x}\|^{2}}\left(2-\frac{\|\bar{x}\|^{2}}{2 \Re\left\{\bar{x}^{H} \boldsymbol{x}\right\}-\|\bar{x}\|^{2}}-\frac{\boldsymbol{y}}{\bar{y}}\right) \\
& \quad-\frac{\bar{t}^{2} \ln \left(1+\|\bar{x}\|^{2} / \bar{y}\right)}{\boldsymbol{t}} \\
& \forall(\boldsymbol{x}, \boldsymbol{y}, \boldsymbol{t}) \in \mathbb{C}^{n} \times \mathbb{R}_{+}^{2},(\bar{x}, \bar{y}, \bar{t}) \in \mathbb{C}^{n} \times \mathbb{R}_{+}^{2},
\end{aligned}
$$

over the trust region

$$
2 \Re\left\{\bar{x}^{H} x\right\}-\|\bar{x}\|^{2}>0 .
$$

\section{REFERENCES}

[1] M. Bennis, M. Debbah, and H. V. Poor, "Ultra reliable and low-latency wireless communication: Tail, risk, and scale," Proc. IEEE, vol. 106, pp. 1834-1853, Oct. 2018.

[2] S. Hong, J. Brand, J. I. Choi, J. Mehlman, S. Katti, and P. Levis, "Application of self-interference cancellation in 5G and beyond," IEEE Commun. Mag., vol. 52, pp. 114-21, Feb. 2014.

[3] Z. Zhang, X. Chai, K. Long, A. V. Vasilakos, and L. Hanzo, "Full duplex techniques for 5G networks: self-interference cancellation, protocol design, and relay selection," IEEE Commun. Mag., vol. 53, no. 5, pp. 128-136, 2015.

[4] Z. Zhang, K. Long, A. V. Vasilakos, and L. Hanzo, "Full-duplex wireless communications: Challenges, solutions, and future research directions," Proc. IEEE, vol. 104, no. 7, pp. 1369-1409, 2016.

[5] A. Sabharwal et al, "In-band full-duplex wireless: challenges and opportunities," IEEE J. Selected Areas in Commun., vol. 32, pp. 1637-1652, Sep 2014. 
[6] M. Heino et al, "Recent advances in antenna design and interference cancelllation algorithms for in-band full duplex relays," IEEE Commun. Magazine, pp. 91-101, May 2015.

[7] D. W. K. Ng, E. S. Lo, and R. Schober, "Dynamic resource allocation in MIMO-OFDMA systems with full-duplex and hybrid relaying," IEEE Trans. Commun., vol. 60, pp. 1291-1303, May 2012.

[8] H. Q. Ngo, H. A. Suraweera, M. Matthaiou, and E. G. Larsson, "Multipair full-duplex relaying with massive arrays and linear processing," IEEE J. Sel. Areas Commun., vol. 32, pp. 1721-1737, 2014.

[9] S. Huberman and T. Le-Ngoc, "MIMO full-duplex precoding: A joint beamforming and self-interference cancellation structure," IEEE Trans. Wirel. Commun., vol. 14, pp. 2205-2217, Apr. 2015.

[10] H. H. M. Tam, H. D. Tuan, and D. T. Ngo, "Successive convex quadratic programming for quality-of-service management in full-duplex MUMIMO multicell networks," IEEE Trans. Comm., vol. 64, pp. 23402353, 2016.

[11] H. H. M. Tam, H. D. Tuan, A. A. Nasir, T. Q. Duong, and H. V. Poor, "MIMO energy harvesting in full-duplex multi-user networks," IEEE Trans. Wirel. Commun., vol. 16, no. 5, pp. 3282-3297, 2017.

[12] H. D. Tuan, D. T. Ngo, and H. H. M. Tam, "Joint power allocation for MIMO-OFDM full-duplex relaying communications," Eurasip J. Wirel. Commun. Networ., 2017, DOI 10.1186/s13638-016-0800-4.

[13] D. Wen, G. Yu, R. Li, Y. Chen, and G. Y. Li, "Results on energy- and spectral-efficiency tradeoff in cellular networks with full-duplex enabled base stations," IEEE Trans. Wirel. Commun., vol. 16, no. 3, pp. 1494 1507,2017

[14] Z. Sheng, H. D. Tuan, H. H. M. Tam, H. H. Nguyen, and Y. Fang, "Energy-efficient precoding in multicell networks with full-duplex base stations," Eurasip J. Wirel. Commun. Network., 2017, DOI 10.1186/s13638-017-0831-5.

[15] B. K. Chalise, H. A. Suraweera, G. Zheng, and G. K. Karagiannidis, "Beamforming optimization for full-duplex wireless-powered MIMO systems," IEEE Trans. Commun., vol. 65, no. 9, pp. 3750-3764, 2017.

[16] X. Quan, Y. Liu, S. Shao, C. Huang, and Y. Tang, "Impacts of phase noise on digital self-interference cancellation in full-duplex communications," IEEE Trans. Signal Process., vol. 65, no. 7, pp. 1881-1893, 2017.

[17] E. Sharma, R. Budhiraja, K. Vasudevan, and L. Hanzo, "Full-duplex massive MIMO multi-pair two-way AF relaying: Energy efficiency optimization," IEEE Trans. Commun., vol. 66, no. 8, pp. 3322-3340, 2018.

[18] T.-H. Chang, Y.-F. Liu, and S.-C. Lin, "QoS-based linear transceiver optimization for full-duplex multiuser communications," IEEE Trans. Signal Process., vol. 66, no. 9, pp. 2300-2313, 2018.

[19] Z. Sheng, H. D. Tuan, T. Q. Duong, H. V. Poor, and Y. Fang, "Lowlatency multiuser two-way wireless relaying for spectral and energy efficiencies," IEEE Trans. Signal Process, vol. 66, no. 16, pp. 43624376, 2018.

[20] A. A. Nasir, H. D. Tuan, D. T. Ngo, T. Q. Duong, and H. V. Poor, "Beamforming design for wireless information and power transfer systems: Receiver power-splitting vs transmit time-switching," IEEE Trans. Commun., vol. 65, no. 2, pp. 876-889, 2017.

[21] A. A. Nasir, H. D. Tuan, and T. Q. Duong, "Fractional time exploitation for serving IoT users with guaranteed QoS by $5 \mathrm{G}$ spectrum," IEEE Commun. Mag., vol. 56, no. 10, pp. 128-133, 2018.

[22] Z. Sheng, H. D. Tuan, T. Q. Duong, and L. Hanzo, "UAV-aided two-way multi-user relaying," under submission to IEEE Trans. Commun.

[23] L. Wang and L. Hanzo, "Optimum time resource allocation for TDMAbased differential decode-and-forward cooperative systems: a capacity perspective," IEEE Commun. Lett., vol. 14, pp. 506-508, Jun. 2010.

[24] L. Wang, L. Li, C. Xu, D. Liang, S. X. Ng, and L. Hanzo, "Multiplesymbol joint signal processing for differentially encoded single- and multi-carrier communications: Principles, designs and applications," IEEE Commun. Surveys \& Tuts., vol. 16, pp. 689-712, 2nd quarter 2014.

[25] S. Buzzi, C.-L. I, T. E. Klein, H. V. Poor, C. Yang, and A. Zappone, "A survey of energy-efficient techniques for $5 \mathrm{G}$ networks and challenges ahead," IEEE J. Select. Areas Commun., vol. 34, pp. 697-709, Apr. 2016.

[26] A. Zappone, L. Sanguinetti, G. Bacci, E. A. Jorswieck, and M. Debbah, "Energy-efficient power control: A look at 5G wireless technologies," IEEE Trans. Signal Process., vol. 64, pp. 1668-1683, Apr. 2016.

[27] M. Duarte, C. Dick, and A. Sabharwal, "Experiment-driven characterization of full-duplex wireless systems," IEEE Trans. Wireless Commun., vol. 11, pp. 4296-4307, Dec. 2012.

[28] M. Duarte, A. Sabharwal, V. Aggarwal, R. Jana, K. Ramakrishnan, C. Rice, and N. Shankaranarayanan, "Design and characterization of a full-duplex multiantenna system for WiFi networks," IEEE Trans. Veh. Technol., vol. 63, pp. 1160-1177, Mar. 2014.

[29] D. Korpi et al, "Full-duplex transceiver system calculations: analysis of adc and linearity challenges," IEEE Trans. Wireless Commun., vol. 13, pp. 3821-3836, Jul 2014.

[30] L. Anttila et al, "Modeling and efficient cancelation of nonlinear selfinterference in MIMO full-duplex transceivers," in Proc. of Globecom, pp. 777-783, Dec. 2014.

[31] C. Xiong, G. Y. Li, S. Zhang, Y. Chen, and S. Xu, "Energy-efficient resource allocation in OFDMA networks," IEEE Trans. Commun., vol. 60, pp. 3767-3778, Dec. 2012.

[32] W. Dinkelbach, "On nonlinear fractional programming," Management Science, vol. 13, pp. 492-498, Jul. 1967.

[33] L. D. Nguyen, H. D. Tuan, T. Q. Duong, O. A. Dobre, and H. V. Poor, "Downlink beamforming for energy-efficient heterogeneous networks with massive MIMO and small cells," IEEE Trans. Wirel. Commun., vol. 17, no. 5, pp. 3386-3400, 2018.

[34] B. R. Marks and G. P. Wright, "A general inner approximation algorithm for nonconvex mathematical programms," Operations Research, vol. 26, pp. 681-683, Jul. 1978.

[35] "3GPP technical specification group radio access network evolved universal terrestrial radio access (E-UTRA): Further advancements for E-UTRA physical layer aspects (release 9)," 2010.

[36] "Channels, physical. "mapping of transport channels onto physical channels (fdd)." gpp technical specification (2003)," 2010.

[37] O. Arnold, F. Richter, G. Fettweis, and O. Blume, "Power consumption modeling of different base station types in heterogeneous cellular networks," in 2010 Future Network \& Mobile Summit, pp. 1-8, IEEE, 2010.

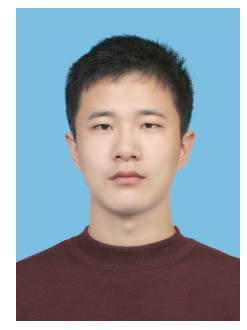

Hongwen Yu received the B.S. degree in Communication and Information Engineering from the Shanghai University, Shanghai, China, in 2011, and the M.S. degree in Communication and Information Engineering from the Shanghai University, Shanghai, China, in 2014. He is currently pursuing the Ph.D. degree with the School of Electrical and Data Engineering, University of Technology Sydney, Ultimo, NSW, Australia, and with Shanghai University, Shanghai, China. His current research interests include optimization methods for wireless communication and signal processing.

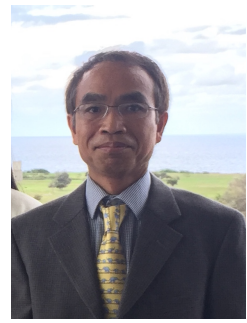

Hoang Duong Tuan received the Diploma (Hons.) and Ph.D. degrees in applied mathematics from Odessa State University, Ukraine, in 1987 and 1991, respectively. He spent nine academic years in Japan as an Assistant Professor in the Department of Electronic-Mechanical Engineering, Nagoya University, from 1994 to 1999, and then as an Associate Professor in the Department of Electrical and Computer Engineering, Toyota Technological Institute, Nagoya, from 1999 to 2003. He was a Professor with the School of Electrical Engineering and Telecommunications, University of New South Wales, from 2003 to 2011. He is currently a Professor with the School of Electrical and Data Engineering, University of Technology Sydney. He has been involved in research with the areas of optimization, control, signal processing, wireless communication, and biomedical engineering for more than 20 years. 


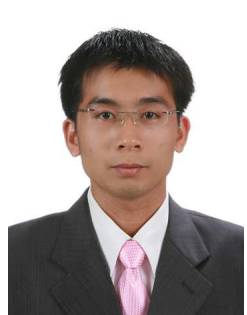

Trung Q. Duong (S'05, M'12, SM'13) received his $\mathrm{Ph} . \mathrm{D}$. degree in Telecommunications Systems from Blekinge Institute of Technology (BTH), Sweden in the end of 2012. Currently, he is a Professor with Queen's University Belfast (UK), where he was a Lecturer (Assistant Professor) (2013-2017), a Reader (Associate Professor) (2018-2020), and Full Professor from August 2020. His current research interests include wireless communications, machine learning, realtime optimisation, big data, and IoT applications to disaster management, airquality monitoring, flood monitoring, smart agriculture, healthcare and smart cities. He is the author or co-author of over $350+$ technical papers published in scientific journals (220+ articles) and presented at international conferences (140+ papers).

Dr. Duong currently serves as an Editor for the IEEE TRANSACTIONS ON Wireless COMmunications, IEEE TRANSACTIONS ON COMMUNICATIONS, and an Executive Editor for IEEE COMMUNICATIONS LETTERS. He was awarded the Best Paper Award at the IEEE Vehicular Technology Conference (VTC-Spring) in 2013, IEEE International Conference on Communications (ICC) 2014, IEEE Global Communications Conference (GLOBECOM) 2016 and 2019, IEEE Digital Signal Processing Conference (DSP) 2017, and International Wireless Communications \& Mobile Computing Conference (IWCMC) 2019. He is the recipient of prestigious Royal Academy of Engineering Research Fellowship (2016-2020) and has won a prestigious Newton Prize 2017

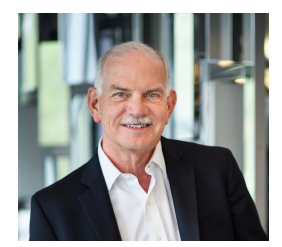

H. Vincent Poor (S72, M77, SM82, F87) received the Ph.D. degree in EECS from Princeton University in 1977. From 1977 until 1990, he was on the faculty of the University of Illinois at Urbana-Champaign. Since 1990 he has been on the faculty at Princeton, where he is currently the Michael Henry Strater University Professor of Electrical Engineering. During 2006 to 2016, he served as Dean of Princeton's School of Engineering and Applied Science. He has also held visiting appointments at several other universities, including most recently at Berkeley and Cambridge. His research interests are in the areas of information theory, signal processing and machine learning, and their applications in wireless networks, energy systems and related fields. Among his publications in these areas is the recent book Multiple Access Techniques for 5G Wireless Networks and Beyond (Springer, 2019).

Dr. Poor is a member of the National Academy of Engineering and the National Academy of Sciences, and is a foreign member of the Chinese Academy of Sciences, the Royal Society, and other national and international academies. Recent recognition of his work includes the 2017 IEEE Alexander Graham Bell Medal and a D.Eng. honoris causa from the University of Waterloo awarded in 2019.

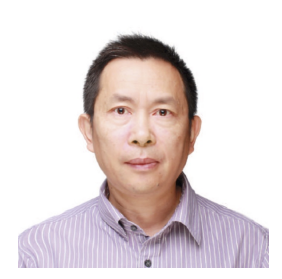

Yong Fang received the Ph.D. degree in Electronic Engineering from City University of Hong Kong, Hong Kong, in 1999. He is now a professor in the School of Communication and Information Engineering, Shanghai University, Shanghai, China. His research interests include communication signal processing, blind signal processing, and adaptive information system. 